Computing and Informatics, Vol. 39, 2020, 105 140, doi: 10.31577/cai_2020_1-2 105

\title{
MULTI-DIMENSIONAL RECOMMENDATION SCHEME FOR SOCIAL NETWORKS CONSIDERING A USER RELATIONSHIP STRENGTH PERSPECTIVE
}

\author{
Bo ZHANG
}

College of Information, Mechanical and Electrical Engineering

Shanghai Normal University, Shanghai 200234, China

E)

Institute of Artificial Intelligence on Education

Shanghai Normal University Shanghai 200234, China

\author{
Ya Zhang, Yanhong BaI, Jie Lian, Meizi Li \\ College of Information, Mechanical and Electrical Engineering \\ Shanghai Normal University, Shanghai 200234, China \\ e-mail: $\{$ lianjie, limeizi\}@shnu.edu.cn
}

\begin{abstract}
Developing a computational method based on user relationship strength for multi-dimensional recommendation is a significant challenge. The traditional recommendation methods have relatively low accuracy because they lack considering information from the perspective of user relationship strength into the recommendation algorithm. User relationship strength reflects the degree of closeness between two users, which can make the recommendation system more efficient between users in pairs. This paper proposes a multi-dimensional comprehensive recommendation method based on user relationship strength. We take three main factors into consideration, including the strength of user relationship, the similarity of entities, and the degree of user interest. First, we introduce a novel method to generate a user candidate set and an entity candidate set by calculating the relationship strength between two users and the similarity between two entities. Then, the algorithm will calculate the user interest degree of each user in the user candidate set to each entity in the entity candidate set, if the user interest degree is larger than or equal to a threshold, this particular entity will be recommended to this user. The performance of the proposed method was verified based on the real-world social network
\end{abstract}


dataset and the e-commerce website dataset, and the experimental result suggests that this method can improve the recommendation accuracy.

Keywords: Recommendation system, social network, user relationship strength, user interest, entity similarity

\section{INTRODUCTION}

Nowadays, as all kinds of social networks (such as Facebook, Twitter, MySpace, etc.) are developing rapidly, these websites have become the major platforms for people's life, work and entertainment. Moreover, recommendation systems have been widely used in many e-commerce websites that can recommend products to target users according to the recommendation algorithms. Generally, the traditional recommendation algorithms ignored the user relationship in social networks, but the fact is that friends tend to have similar shopping preferences, and consumers may purchase a product based on what their friends purchased as well. Therefore, the traditional recommendation algorithms have relatively low accuracy in practical applications.

In recent years, many studies have addressed how to connect social networks to e-commerce websites in recommendation algorithms. The traditional recommendation algorithms mainly include the collaborative filtering recommendation algorithm [1, 2, 3], the content-based recommendation algorithm [4, 5, 6] and the knowledge-based recommendation algorithm [7, 8, 9], etc. In the collaborative filtering recommendation model, when the collaborative filtering recommendation algorithm is based on similar users, its performance is bad. And when the algorithm is based on similar entities, it results in some problems, such as data sparseness and cold start. In the content-based recommendation model, the recommendation results show that it is not ideal for unstructured information. In the knowledgebased recommendation model, the additional information that needs to be provided manually is required, and the information is not only difficult to obtain but also expensive. Therefore, from the perspective of user relationship strength, we suggest that more recommendation factors should be comprehensively considered in the recommendation system in order to achieve consumer satisfaction and maximize business profits.

Based on this analysis, we propose a novel multi-dimensional comprehensive recommendation method based on the social network. First, we present three algorithms to calculate user tightness, user interest degree and entity similarity, respectively. These algorithms are developed according to the social network analysis, such as the interaction frequency between users, comment stability, and similar communities. Then, an entity candidate set is generated based on the entities in the e-commerce website, and a user candidate set is generated based on the users in the social network. After that, the novel recommendation algorithm will recommend 
entities from the entity candidate set to the target user. The recommended entity must satisfy certain conditions by using the correlation algorithm to the users in the user candidate set. To the best of our knowledge, considering both the entity similarity dimension, friend's tightness dimension and user interest dimension in recommendation methods was rarely studied before. And since the social network characteristics will affect users' purchase motivation to some extent, considering social network factors in the recommendation method can improve the recommendation accuracy.

In general, our work aims at improving the recommendation performance by providing a novel recommendation algorithm that can take both the user relationship, entity similarity and user interest degree into consideration. The main contributions are summarized as follows:

1. Three methods were introduced to define and estimate the tightness between users, the similarity between entities, and the user interest degree, respectively, by considering the comments stability between users, friend reliability, interaction frequency, mutual neighbors and similar communities, and some entity attributes.

2. A novel multi-dimensional comprehensive recommendation method based on user tightness, entity similarity, and user interest degree was proposed to recommend entities to the target users from the perspective of user relationship strength.

The rest of the paper is organized as follows: Section 2 reviews the literature related to this study, Section 3 presents the problem definition, Section 4 introduces the multi-dimensional comprehensive recommendation method, Section 5 presents the experimental results, and Section 6 concludes this study and provides some future suggestions.

\section{RELATED WORKS}

Recommendation system first introduced by Resnick and Varian [10] can provide product suggestions for users when users do online shopping based on information retrieval and information filtering. In general, the recommendation system contains three elements, including entities, users, and recommendation algorithm. According to different algorithms, recommendation systems can be divided into four types, which contain content-based recommendation systems, collaborative filtering recommendation systems, knowledge-based recommendation systems, and hybrid recommendation systems [11, 12].

A content-based recommendation system needs to calculate user similarities based on their historical purchase records, and extract user characteristics by statistics and machine learning methods. This system has been applied in many areas. For example, Puglisi et al. [13] proposed a content-based recommendation method and user privacy technique in social-tagging systems. Musto et al. [14] proposed a rec- 
ommendation system by learning word embeddings from Wikipedia. Gu et al. [15] introduced a method by learning global term weights to the content-based recommendation system. In general, the content-based recommendation system can be used to deal with structured information (news and articles) well. However, for the unstructured information, it has a relatively low performance.

Recently, the collaborative filtering recommendation method has become one of the most successful methods that can realize personalized services. This method needs to calculate the similarity between the target user and the other users. And users with a bigger similarity tend to purchase similar products. The collaborative filtering method has been applied in many systems, such as joke recommendation [16], news recommendation [17] and movie recommendation [18]. Additionally, Fang et al. 19 proposed a generalized cross-domain collaborative filtering framework that can integrate social network information seamlessly with cross-domain data. Du et al. 20] developed a method based on the trust network that can improve the system performance greatly. Although the collaborative filtering method has been widely used, there are still some problems that need to be solved, such as data sparsity and scalability.

The knowledge-based recommendation system usually needs to use additional information about the current user and effective entities based on knowledge. This kind of system is often applied to specific areas, such as e-learning recommendation [21], music recommendation [22], and e-commerce product recommendation [23]. The major advantage of the knowledge-based recommendation system is that it can avoid the cold start problem because it does not need to rely on user information to calculate the product entity scores [24, 25].

The hybrid recommendation system employs a new algorithm that can combine the above three recommendation algorithms. For example, Wang et al. [26] proposed a hybrid recommendation model that contains two key components: incremental update item-based collaborative filtering and latent semantic analysis based relative term frequency algorithms. Zhu et al. 27] proposed a hybrid model combining the collaborative filtering algorithm with the knowledge map to represent the learning method, which can improve the recommendation performance greatly.

However, the previous methods still have some problems. First, most of the recommendation methods took only one factor into consideration, such as only the similarity between users, the similarity between entities, and the user's interest to the entity. Second, the traditional recommendation methods cannot be applied to social networks, which will affect the recommendation accuracy. In order to solve the above problems, in this study, we propose a multi-dimensional comprehensive recommendation method based on user relationship strength in social networks. This method considers and quantifies the tightness between users, the user explicit interest to entities, and the entity similarity, which can improve the recommendation performance in accuracy, coverage, and the recommendation diversification compared with traditional recommendation systems. 


\section{PROBLEM DEFINITION}

The social network is a graph model that can describe the relationship between users. The vertexes in the graph model represent users, and the edges between vertexes represent the user relationship. The binary relation of social network graph can describe the relationship between users, which is consistent with the social connection between people in real life. Since a user and his (her) friends are most likely to have similar interests, prediction of user's preferences based on his (her) friends' interests is often used in the recommendation system nowadays. The relevant definitions are described below.

Definition 1 (Community Model). A community $C=\langle C V, C E\rangle$ is a sub-graph of the social network, and it is composed of users who have similar interests, where $C V \subseteq V, C E \subseteq E$.

Definition 2 (Tightness of Users). The tightness of two users reflects the closeness degree between them. The frequent contacts between the source user and target user normally represent that they trust each other, which means the link between them is stable. Therefore, to compute the tightness of users, five aspects are considered in this paper, including the comments stability, the friend reliability, the interaction frequency, the mutual neighbors and the similar communities. The tightness of users is denoted by closeness $(s u, t u)$, where $s u$ represents the source user, and $t u$ represents the target user.

Definition 3 (User Interest Degree). User interest degree $I(v \mid$ item) reflects the interested level that the user $v$ is to the entity item. It is commonly used to predict the purchase probability of a user to a particular entity. The user interest can be divided into explicit interest and implicit interest. Explicit interest can be expressed directly by the users' behaviors, such as commenting, browsing time, forwarding, and approving. The implicit interest means that the user may purchase the products in the same category as the product he (she) purchased before.

Definition 4 (Entity Similarity). The entity similarity $\operatorname{sim}\left(\right.$ item $_{k}$, item $\left._{j}\right)$ describes the degree of consistency between two entities, where item $_{k}$ denotes the entity $k$ and item $_{j}$ denotes the entity $j$. The entity similarity is calculated based on four attributes, including category, price, quality, and discount.

With the notations introduced above in Table 1, we define our recommendation problem as follows. Given an e-commerce website, let user $u_{i} \in U=\left\{u_{1}, u_{2}, \ldots, u_{n}\right\}$, where $U$ denotes a set of users, the friends set of user $u_{i}$ is denoted by friend $\left(u_{i}\right)=$ $\left\{\right.$ friend $_{1}$, friend $_{2}, \ldots$, friend $\left._{x}\right\}$. Now, if a user $u_{i}$ has already purchased an entity item $_{j} \in$ Item $=\left\{\right.$ item $_{1}$, item $_{2}, \ldots$, item $\left._{m}\right\}$, then some entities from Item will be recommended to $u_{i}$ 's friends from the friends set friend $\left(u_{i}\right)$, based on the tightness of users closeness $(s u, t u)$, entity similarity $\operatorname{sim}\left(\operatorname{item}_{k}\right.$, item $\left._{j}\right)$ and user interest degree $I(v \mid$ item $)$. 


\begin{tabular}{l|l}
\hline Algebraic Symbol & Description \\
\hline$C$ & The community is composed of users with similar interests \\
\hline$C=\langle C V, C E\rangle$ & A sub-graph of social network \\
\hline$C V \subseteq V$ & $\begin{array}{l}\text { The users in the community belong to users in the social } \\
\text { network }\end{array}$ \\
\hline$C E \subseteq E$ & $\begin{array}{l}\text { The edges between users in the community belong to the edges } \\
\text { in the social network }\end{array}$ \\
\hline$s u$ & The source user \\
\hline$t u$ & The target user \\
\hline closeness $(s u, t u)_{\text {item }_{k}}$ & The tightness strength between user $s u$ and user $t u$ \\
\hline item $_{j}$ & Entity $k$ \\
\hline $\operatorname{sim}\left(\right.$ item $_{k}$, item $\left._{j}\right)$ & Entity $j$ \\
\hline$I(v \mid$ item $))$ & The similarity of two entities \\
\hline
\end{tabular}

Table 1. Algebraic symbols corresponding to the description

The architecture of our proposed model is shown in Figure 1. The social network consists of a large number of users and user relationships, expressed by a graph model which is the basis of the multidimensional comprehensive recommendation algorithm based on user relationship strength proposed in this paper. We need to complete the calculation of the user relationship strength in this research on the basis of social networks, and it is crucial for the establishment of our recommendation model. The core recommendation algorithm module is comprised of three sub-modules $M_{1}, M_{2}$, and $M_{3}$. The sub-module $M_{1}$ represents the modeling and analysis of user relationship strength. In this sub-module, if the relationship strength between user $u_{i}$ and $u_{i}$ 's friend friend ${ }_{y}$, denoted by closeness $\left(u_{i}\right.$, friend $\left.{ }_{y}\right)$, is larger than or equal to a threshold $\gamma$, then the user friend ${ }_{y}$ will be added to the user candidate set $\mathrm{R}_{-}$user. The sub-module $M_{2}$ represents the modeling and analysis of entity similarity between entities. In this sub-module, if the user $u_{i}$ has purchased an entity item $_{j}$, then calculate the entity similarity $\operatorname{sim}\left(\right.$ item $_{k}$, item $\left._{j}\right)$ between the item $_{j}$ and item $_{k} \in$ Item. And if the similarity is larger than or equal to the threshold $\alpha$, this particular entity item $_{k}$ will be added to the entity candidate set $\mathrm{R}$ _item. The sub-module $M_{3}$ represents the modeling and analysis of how the users in the user candidate set are interested in the entities in the entity candidate set. If the interest degree of the user $u_{i} \in \mathrm{R}_{-}$user to the entity item $_{k} \in \mathrm{R}$ _item is larger than or equal to the threshold $\beta$, then the entity item ${ }_{k}$ will be recommended to the user $u_{i}$.

Obviously, the core modules of the multi-dimensional comprehensive recommendation method proposed in this study are the sub-models $M_{1}, M_{2}$, and $M_{3}$, which can calculate the user relationship strength, the entity similarity, and the user interest degree, respectively. Therefore, the three sub-modules will be introduced in detail in the next section. 


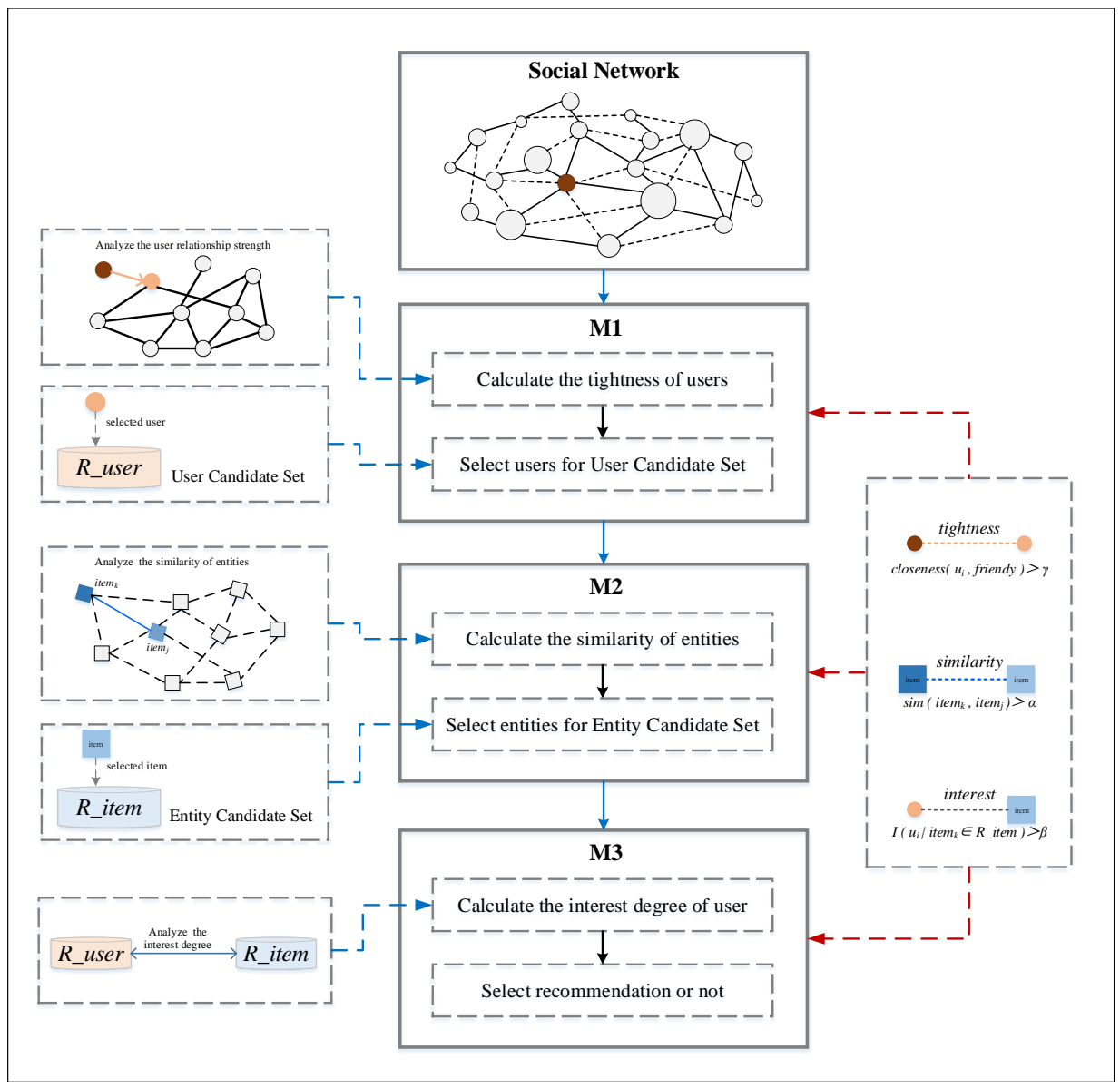

Figure 1. The architecture of the multi-dimensional comprehensive recommendation method

\section{METHOD}

\subsection{Tightness of Users in Social Network}

The connection between users contains direct connection and indirect connection in the social network graph model, whereas only the direct connection is considered in this study. The strength degree of a link reflects the closeness between the two users [28]. A bigger strength degree of a link indicates there is more frequent contact between the source user and the target user, which means they trust each other in a stable way [29]. Normally, four aspects between users are considered to calculate the user tightness, including the comments stability, the reliability of users, the 
frequency of interaction, and mutual neighbors and similar communities [30]. It is shown in Figure 2, where the su represents the source user extracting from the social network, and $t u$ represents the target user that is connected directly with the source user. For example, assume that there is a source user $s u$ whose target user $t u$ is from the set of users directly connected to $s u$. To calculate the user tightness between $s u$ and $t u$, firstly, the scores of COM_STA $(s u, t u), \operatorname{MUT} \_R E L(s u, t u), \operatorname{INT} \_F R E(s u, t u)$ and C_nei-com $(s u, t u)$ need to be calculated separately, then the score of the user tightness is calculated based on these four scores.

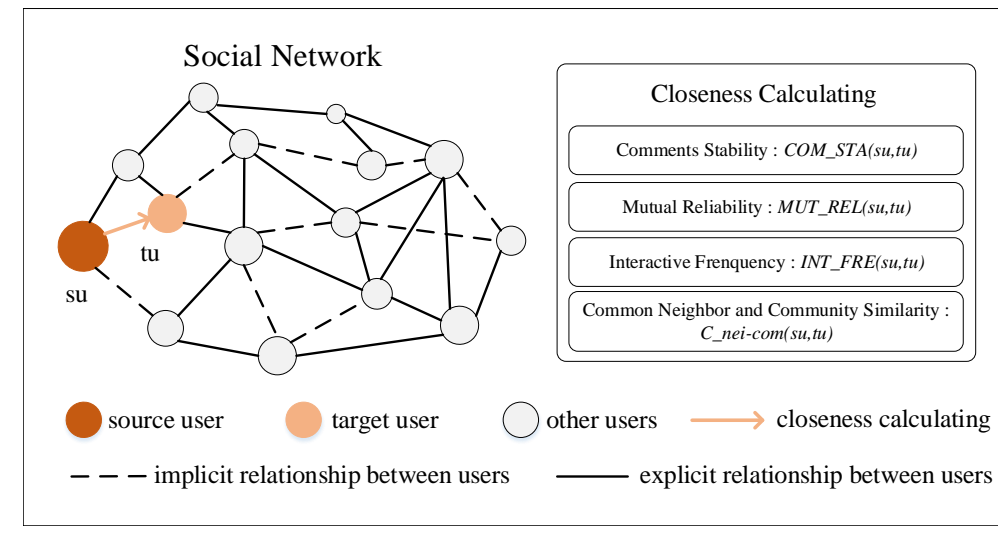

Figure 2. Four dimensions when calculating the user relationship strength

\subsubsection{Comment Stability}

The comment stability reflects the comment fluctuations from the source user to the target user. Many studies have found that higher comment stability indicates the more similar comments of the source user to the target user, and vice versa 31. Therefore, the stability of the comments can reflect the tightness between users. The comments set between the user $s u$ and $t u$ is represented by $\operatorname{COM}(s u, t u)$, the total number of comments set is represented by $|\operatorname{COM}(s u, t u)|$, and the average number of comments is represented by $\overline{\mathrm{com}}(s u, t u)$. Therefore, the comments stability between the source user $s u$ and the target user $t u$ can be calculated by the following equation:

$$
\operatorname{COM} \_S T A(s u, t u)=1-\sqrt{\frac{\sum_{i=1}^{|\operatorname{COM}(s u, t u)|}\left[\operatorname{com}(t u, s u)_{i}-\overline{\operatorname{com}}(s u, t u)\right]^{2}}{|\operatorname{COM}(s u, t u)|}} \text {. }
$$

For example, a user $a$ sends comments to a user $b$, and the comments set is $\operatorname{COM}(a, b)=\{1,1,2,3,3\}$, the average number of comments is represented by $\overline{\operatorname{com}}(a, b)=2$. Assume that the user $b$ gives comments to the user $a$, and the comments set is $\operatorname{COM}(b, a)=\{2,1,3,2,2\}$, then the comments stability value between 
the source user $a$ and the target user $b$ can be calculated as follows,

$$
\begin{aligned}
& \text { COM_STA }(a, b)=1-\sqrt{\frac{(2-2)^{2}+(1-2)^{2}+(3-2)^{2}+(2-2)^{2}+(2-2)^{2}}{5}} \\
& \approx 0.3675 \text {. }
\end{aligned}
$$

\subsubsection{Mutual Reliability Degree}

The mutual reliability degree represents the reliability between two users, and it is reflected by three aspects: reliabilities of the comment, forwarding and approving. In this paper, given two users that are connected directly, the reliability degree considering all three factors can be denoted by

$$
\begin{gathered}
R_{-} C\left(u_{1}, u_{2}\right)=\left\{v_{1}=\text { reliability_com }\left(u_{1}, u_{2}\right), v_{2}=\text { relibility_for }\left(u_{1}, u_{2}\right)\right. \\
\left.v_{3}=\text { reliability_apr }\left(u_{1}, u_{2}\right)\right\}
\end{gathered}
$$

where $v_{1}$ represents the reliability of the comments, $v_{2}$ represents the forwarding reliability and $v_{3}$ represents the approving reliability. Then the mutual reliability (denoted by MUT_REL(su,tu)) can be calculated by Equation (3).

$$
\operatorname{MUT} \_\operatorname{REL}(s u, t u)=\frac{\sum_{i=1}^{3} R_{-} C(s u, t u) \cdot v_{i} \times R_{-} C(t u, s u) \cdot v_{i}}{\sqrt{\sum_{i=1}^{3}\left(R_{-} C(s u, t u) \cdot v_{i}\right)^{2}} \times \sqrt{\sum_{i=1}^{3}\left(R_{-} C(t u, s u) \cdot v_{i}\right)^{2}}} .
$$

For instance, there are user $a$ and user $b$, and the reliability degree $R_{-} C(a, b)$ from $a$ to $b$ is 0.7 , reliability degree $R_{-} C(b, a)$ from $b$ to $a$ is 0.6 . Assume that $v_{1}, v_{2}$ and $v_{3}$ of $R_{-} C(a, b)$ are $0.6,0.7,0.5$, and the $v_{1}, v_{2}$ and $v_{3}$ of $R_{-} C(b, a)$ are 0.5, 0.6, 0.3 , respectively. Then, the mutual reliability can be calculated as follows:

$$
\begin{aligned}
\operatorname{MUT} \_\operatorname{REL}(a, b) & =\frac{(0.7 \times 0.6 \times 0.6 \times 0.5)+(0.7 \times 0.7 \times 0.6 \times 0.6)+(0.7 \times 0.5 \times 0.6 \times 0.3)}{\sqrt{(0.7 \times 0.6)^{2}+(0.7 \times 0.7)^{2}+(0.7 \times 0.5)^{2}} \times \sqrt{(0.6 \times 0.5)^{2}+(0.6 \times 0.6)^{2}+(0.6 \times 0.3)^{2}}} \\
& \approx 0.6750 .
\end{aligned}
$$

\subsubsection{Interactive Frequency}

Interaction frequency can signify the relationship between users in social networks. To retrieve the degree of interactive frequency, three indicators will be used, including the number of interactions in unit time, the average time length of interaction, and the average interaction time interval.

For the source user su, assume that the maximum number of interactions between this user and the user's friends is $\operatorname{MAX}[\operatorname{num}(s u)]_{u k}$ in unit time $u_{k}$. Among all these interactions, the longest time length of a continuous interaction is denoted by MAX $[\operatorname{len}(s u)]_{u k}$, and the shortest time interval of these interactions is denoted by 
$\operatorname{MIN}[\operatorname{int}(s u)]_{u k}$. The numbers of the past interactions from the source user to the target user is denoted by $\operatorname{NUM}(s u, t u)_{u k}$ in unit time $u_{k}$. The average time length of the past interactions from the source user to the target user is denoted by $\operatorname{LEN}(s u, t u)_{u k}$, and the average time interval of the past interactions is denoted by $\operatorname{INT}(s u, t u)_{u k}$. Then, in this study, the interaction frequency denoted by INT_FRE(su,tu) can be calculated by Equation (4):

$$
\operatorname{INT} \_ \text {FRE }(s u, t u)=\frac{\sum_{k=1}^{|\mathrm{Unit}|}\left(l_{k}\right)}{\mid \text { Unit } \mid}
$$

where |Unit| is the number of time units, and $l_{k}$ is the value of interaction frequency factors in unit time $u_{k}$, which can be calculated by Equation (5):

$$
l_{k}=\frac{1}{3}\left[\frac{\operatorname{NUM}(s u, t u)_{u k}}{\operatorname{MAX}[\operatorname{NUM}(s u)]_{u k}}+\frac{\operatorname{LEN}(s u, t u)_{u k}}{\operatorname{MAX}[L E N(s u)]_{u k}}+\frac{\operatorname{MIN}\left[\operatorname{INT}(s u)_{u k}\right]}{\operatorname{INT}(s u, t u)_{u k}}\right] .
$$

Assume that there are user $a$ and user $b$, and let the maximum number of interaction, the longest time length of continuous interaction, and the shortest time interval of the interaction be 8 minutes, 25 minutes, and 5 minutes in this example, respectively. If the number of the past interaction from $a$ to $b$ is 5 , and let the average time length and the average time interval of the past interactions be 20 minutes and 15 minutes, respectively, then the $l_{k}$ is $\frac{1}{3}\left(\frac{5}{7}+\frac{20}{25}+\frac{15}{5}\right)$. Next, we assume the total number of user interaction time units $\mid$ Unit $\mid$ is 5 , and the values of interaction frequency factors $l_{k}$ in these unit times are 1.3, 1.1, 0.6, 0.7, and 0.5, respectively. Then the interaction frequency can be calculated by

$$
\operatorname{INT} \_F R E(a, b)=\frac{1.3+1.1+0.6+0.7+0.5}{5}=0.84 .
$$

\subsubsection{Common Neighbor and Similar Community}

Since community can promote interactions between users, common neighbors and similar communities are used to evaluate the link strength between users in this study. First, considering similar community, customer intimacy is decreasing as the growth of the community scale, which means the smaller size community will bring more contributions than the larger community. Second, the number of common neighbors also reflects the link intensity between two users. That is to say, two users who have more common neighbors will generate a stronger relationship between them.

The source user community is denoted by $C_{s u}$ and the target user community is denoted by $C_{t u}$. Then, the intersection between the source user community and the target user community is denoted by Same $C_{i} \in C_{s u} \cap C_{t u}$, in which the source user and the target user have their neighbors set expressed by $N_{\mathrm{Same}_{i}}(s u)$ and $N_{\mathrm{Same}_{i}}(t u)$, respectively. Here, we use $\left|\mathrm{Same}_{i}\right|$ and $\left|\mathrm{Same}_{i}(s u)\right|$ to express the number of members in the community $S_{a m e} C_{i}$, and the number of neighbors in 
the set $\mathrm{Same}_{i}(s u)$, respectively. Therefore, the common neighbors and similar community expressed as C_nei-com $(s u, t u)$ can be calculated by Equation (6) as follows:

$$
\text { C_nei-com }(s u, t u)=\frac{\sum_{N_{\mathrm{Same}} C_{i} \in C_{s u} \cap C_{t u}}\left[\left(\frac{1}{\log _{2}\left|\operatorname{Same} C_{i}\right|}\right) \times\left|\frac{N_{\mathrm{SameC}_{i}}(s u) \cap N_{\mathrm{Same}_{i}}(t u)}{N_{\mathrm{Same}_{i}}(s u) \cup N_{\mathrm{SameC}_{i}}(t u)}\right|\right]}{\sum_{N_{\mathrm{Same}} C_{i} \in C_{s u} \cap C_{t u}}\left(\frac{1}{\log _{2}\left|\operatorname{Same} C_{i}\right|}\right)} .
$$

For example, to make it easier to understand, we assume that the number of intersection communities between the source user and the target user is 2, i.e. $\mid$ Same $C_{i} \mid=2$. Meanwhile, we suppose that the number of the intersection between the source user's neighbors set and the target user's neighbors set in these two common communities are 12 and 16, and the number of the union between those neighbors set are 20 and 20, respectively. Then, the common neighbors and similar community can be calculated as follows:

$$
\text { C_nei-com }(s u, t u)=\frac{\left[\left(\frac{1}{\log _{2} 2}\right) \times\left|\frac{12}{20}\right|\right]+\left[\left(\frac{1}{\log _{2} 2}\right) \times\left|\frac{16}{20}\right|\right]}{\frac{1}{\log _{2} 2}+\frac{1}{\log _{2} 2}}=0.7 \text {. }
$$

At last, according to the community stability COM_STA $(s u, t u)$, the mutual reliability degree MUT_REL $(s u, t u)$, the interactive frequency INT_FRE $(s u, t u)$, and the common neighbors and similar community C_nei-com $(s u, t u)$, we can calculate the link strength between users, denoted by closeness $(s u, t u)$ in Equation (7):

$$
\begin{aligned}
\operatorname{closeness}(s u, t u)= & \frac{1}{4}\left[\mathrm{COM} \_\mathrm{STA}(s u, t u)+\operatorname{MUT} \_R E L(s u, t u)\right. \\
& \left.+\operatorname{INT} \_\mathrm{FRE}(s u, t u)+\text { C_nei-com }(s u, t u)\right] .
\end{aligned}
$$

\subsection{Entity Similarity}

Entity similarity is the similarity degree between two entities on the same attribute, which is an important consideration when designing a complete entity recommendation system. Entities contain various attributes, such as price, category, quality, discount, size, color, etc. The attributes of an entity can uniquely identify the corresponding entity. Normally, four attributes of an entity are considered to calculate the entity similarity, including entity category, price, quality, and discount [32]. In this section, we will introduce how to calculate the similarity degree of two entities, denoted by $\operatorname{sim}\left(\right.$ item $_{k}$, item $\left._{j}\right)$, where item represents entity $k$ and item $_{j}$ represents entity $j$. Both the entity $k$ and entity $j$ are from the same entity library, shown in Figure 3. The calculation of the similarity degree between the entity item $_{k}$ and the entity item $_{j}$ is based on the entity category, price, quality, and discount. The calculation equation is in below:

$$
\operatorname{sim}\left(\text { item }_{k}, \text { item }_{j}\right)=\frac{1}{4} \times(\text { sim_type }+ \text { sim_price }+ \text { sim_quality }+ \text { sim_sale })
$$


where sim_type, sim_price, sim_quality and sim_sale represent the type similarity, the price similarity, the quality similarity and the sale similarity, respectively. If the similarity degree between the entity item $_{k}$ and the entity item $_{j}$ is larger than or equal to a threshold $\alpha$, and the value of threshold $\alpha$ is mainly based on the experiment in Section 5 to obtain the optimal solution, then the entities should be put into the entity candidate set R_item. In the following sub-sections, we will introduce how to calculate sim_type, sim_price, sim_quality and sim_sale, respectively.

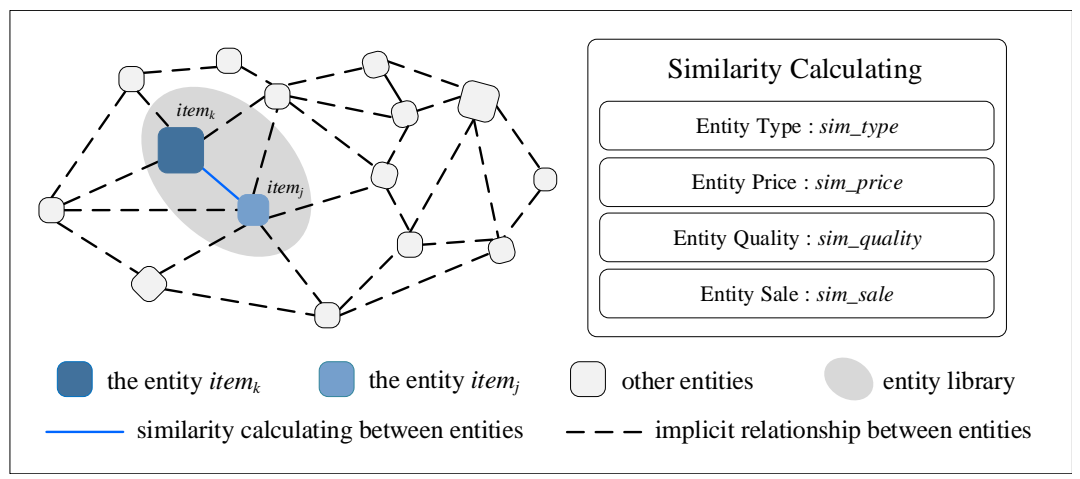

Figure 3. Four dimensions when calculating the entity similarity

\subsubsection{The Calculation of Entity Type Similarity Degree}

Normally, customers will search for products based on the category name, which makes the entity category become the primary consideration in the entity recommendation system. Therefore, in this paper, we propose a method based on tree structure to calculate the similarity degree of the entity type.

The tree structure with $n$ nodes is a hierarchical data structure that is defined with branch relation. In any non-empty tree structure, only one specific node can become the root node. And if there is more than one node in the tree $(n>1)$, then the rest of nodes can be divided into $m(m>0)$ mutually disjoint finite sets, $T_{1}$, $T_{2}, \ldots, T_{m}$, and every set itself is a tree structure called subtree. For example, in Figure $4 \mathrm{a}$ ), there is a tree with only one node; in Figure $4 \mathrm{~b}$ ), there is a tree with 13 nodes, and among which, $A$ is the root node, the rest are divided into three mutually disjoint subtrees $T_{1}=\{B, E, F, K, L\}, T_{2}=\{C, G\}$ and $T_{3}=\{D, H, I, J, M\}$. For the subtree $T_{1}$, the root node is $B$, and the rest four nodes are divided into two mutually disjoint subtrees again, which are $T_{11}=\{E, K, L\}$ and $T_{12}=\{F\}$.

A subtree is a child node of its root node, the root node is called the child node's parent. The child nodes with the same parent are brother nodes, such as node $K$ and node $L$ are brothers in Figure $4 \mathrm{~b}$ ). The ancestors of a node are the nodes that traverse from the root to itself. For example, in Figure $4 \mathrm{~b}$ ), the nodes $A$ and $C$ are ancestors of the node $G$. The level in the tree structure means that the root node 


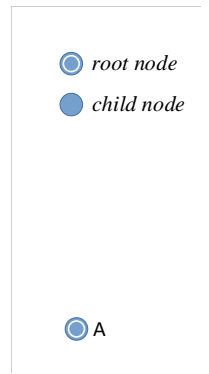

a)

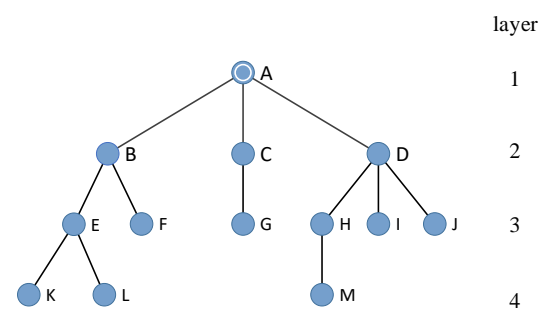

b)

Figure 4. The tree structure: a) tree without child node, b) tree with multiple child nodes

is in the first layer, the children of the root node are in the second layer. And if one node is on the layer $l$, the subtrees of this node are on the layer $l+1$. If the parent nodes of two particular nodes are on the same layer, then these two nodes are cousins. For example, node $E$ and node $G$ are cousins, which is because their parent nodes $B$ and $C$ are on the same layer.

In this paper, we build a tree structure to calculate the type similarity degree of two entities. In this tree structure, entities within the same category will be in the same subtree. The concept of the layer is introduced to distinguish which layer the entity belongs, $c_{i}$ means the category on the layer $i$, where $i \leq 4$, such that $c_{1}$ represents the first entity category. The distanace $\left(\right.$ item $_{x}$, item $\left._{y}\right)$ means the category

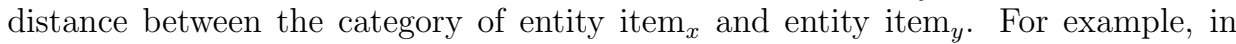
Figure 5, the distance between the entity $\operatorname{item}_{a}$ and entity $\operatorname{item}_{b}$ is 2 .

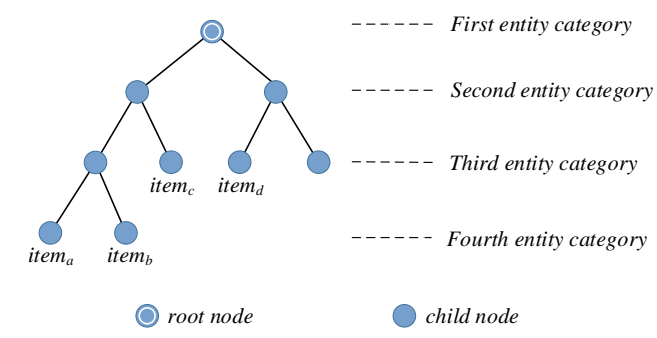

Figure 5. An example of the entity category

When calculating the similarity degree of the entity category (denoted by sim_type), it is required to consider if the categories of these two entities are on the same layer. If the categories of two entities belong to the same layer, then the 
similarity degree calculation equation of the entity category is shown below.

$$
\text { sim_type }=\sqrt[c_{i}]{\frac{1}{1+\operatorname{distance}\left(\text { item }_{x}, \text { item }_{y}\right)}}
$$

where $c_{i}$ represents the layer of the category, distance $\left(\right.$ item $_{x}$, item $\left._{y}\right)$ means the cat-

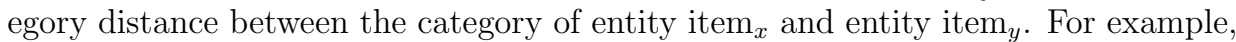
the distance between item ${ }_{c}$ and item $_{d}$ is $4, c_{i}$ is 2 in Figure 5 , so the $\operatorname{sim}_{\text {type }}$ is $\sqrt[2]{\frac{1}{5}}$.

If the categories of the two entities are not in the same layer, then the similarity degree calculation equation of the entity category is shown below.

$$
\text { sim_type }=\sqrt[\frac{c_{x}+c_{y}}{2}]{\frac{1}{1+\operatorname{distance}\left(\text { item }_{x}, \text { item }_{y}\right)}} .
$$

In Figure 5, the distance between item $_{b}$ and item ${ }_{c}$ is $3, c_{b}$ is $3, c_{c}$ is 2 , so the sim_type is $\sqrt[\frac{5}{2}]{\frac{1}{4}}$. And the value range of sim_type is from 0 to 1 .

\subsubsection{The Calculation of Entity Price Similarity Degree}

Consumers will take price as an important consideration when purchasing products. Therefore, the price similarity between two entities will be one important factor of entity similarity. To calculate the price similarity, the entity price will be converted into the elasticity coefficient, then calculate the entity similarity according to the price range. For example, $39=0.39 \times 10^{2}$, if the price range is in $0.39 \times 10^{2} \pm 0.39 \times 10^{1}$, it is the effective candidate entity; otherwise, it is not the effective candidate entity, assuming that the effective candidate entities are denoted by $R_{-} P\left\{\right.$ item $_{1}$, item $_{2}$, item $\left._{3}, \ldots\right\}$. The calculation of sim_price is shown in Equation (11).

$$
\text { sim_price }_{-}=1-\frac{\mid \text { price }_{a}-\text { price }_{b} \mid}{\text { price }_{a}}
$$

where price ${ }_{a}$ is the price of the selected entity item ${ }_{a}$, and price $_{b}$ is the price of the candidate entity item $_{b}$, which is from the entity candidate set. The value range of sim_price is from 0 to 1 as well.

\subsubsection{The Calculation of Entity Quality Similarity Degree}

Entity quality is another important consideration to make entities recommendation. The entity quality similarity, denoted by sim_quality is calculated based on user evaluation according to our common sense. The comment score in total is denoted by max, and the comment score in average is denoted by ave. If the comment score of an entity is equal or greater than ave/max, then put this entity into the recommended candidate set. The equation to calculate the sim_quality is below.

$$
\text { sim_quality }=\frac{\text { score }_{k}}{\max } \geq \frac{\text { ave }}{\max }
$$


where score $_{k}$ is the comment score of the selected entity. The value range of sim_quality is from 0.8 to 1 according to the value of ave/max calculated based on the available data.

\subsubsection{The Calculation of Entity Discount Similarity Degree}

Since consumers will consider if an entity is on sale when making a purchase, the discount similarity between two entities will also be introduced as a factor in entities recommendation system. When calculating the discount similarity between two entities denoted by sim_sale, the discount degree of the target entity is written by sale, if the discount degree of the selected entity is greater than sale and sale is greater than zero, then put this particular entity into the recommended candidate set. The calculation equation of sim_sale is shown as follows:

$$
\text { sim_sale }=\frac{\text { sale_k }- \text { sale }}{\text { sale_k }}
$$

where sale_k is the discount degree of the selected entity and the value range of sim_sale is from 0 to 1 as well.

\subsection{The User Interest Degree}

The user interest degree reflects how a user is interested in an entity, and it can be quantified by a value to predict the purchase probability of an entity [33. The user interest can be divided into explicit interest and implicit interest. The explicit interest can be expressed directly by users' behaviors, such as commenting, browsing frequency, forwarding, and approving [34]. The implicit interest degree is mainly extracted and analyzed by user's relationship because the link relations between users can show possible implicit interest, which was introduced in Section 4.1. It is obvious that the explicit interest can be evaluated through the users' behavior directly, while the implicit interest needs to be extracted by the user relationship. Therefore, only the explicit interest is considered in this section.

In this study, the direct behaviors made by users are used as interest evidence to calculate the explicit interest degree, including forwarding, approving, following and comments. Then, to calculate the user interest degree by interest evidence, we need to consider two aspects. First, the explicit interest is measured by the level and weight of the interest evidence, and each interest evidence will have a different effect on the user interest degree. Second, the explicit interest is affected by the influence degree of the entities, which means users will be more interested in the entity that has a bigger influence.

Taking these two considerations into account, the calculation of the explicit interest degree of entities is introduced below. Assume that the interest evidence from the previous behaviors of the user $v$ is denoted by $D i e_{j} \in\left\{D i e_{1}, D i e_{2}, \ldots, D i e_{m}\right\}$, and $P\left(D i e_{j}\right)$ denotes the frequency of interest evidence $D i e_{j}$. If the user does $n$ interest evidences to an entity, then the interest evidence set can be expressed by 
$I E=\left\{\varsigma_{1}, \varsigma_{2}, \ldots, \varsigma_{i}, \ldots\right\}$; and $P\left(\right.$ item $\mid$ Die $\left._{j}\right)$ indicates the probability of $D i e_{j}$ in the interest evidence of an entity item. The weight of each interest evidence $D_{i e_{j}}$ is denoted by $\operatorname{right}\left(D i e_{j}\right)\left(\operatorname{right}\left(D i e_{j}\right) \in[0,1]\right)$. Then, the explicit interest value about the user node $v$ to an entity is calculated by:

$$
\begin{aligned}
& I(v \mid \text { item }) \\
& = \begin{cases}0, & n=0, \\
{\left[\frac{1}{n} \times \sum_{i=1}^{n}\left(\operatorname{right}_{\varsigma_{i} \in \text { Die }_{j}}\left(\text { Die }_{j}\right) \times P_{v}\left(\text { Die }_{j} \mid \text { item }\right)\right)\right] \times \lambda^{\text {impact }},} & n \geq 1,\end{cases}
\end{aligned}
$$

where $\lambda^{\text {impact }}$ is introduced to represent the influence of explicit interest except implicit interests, and $P_{v}\left(D_{i e_{j}} \mid\right.$ item $)$ denotes the occurrence probability of the interest evidence $D_{i e_{j}}$ for the entity item, and it is calculated as follows:

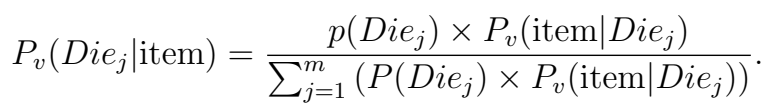

For instance, there are two evidences, i.e. $m=2$, and the values of $P_{v}\left(\operatorname{Die}_{1}\right)$, $P_{v}\left(D_{i e_{2}}\right), P_{v}\left(\right.$ item $\left.\mid D i e_{1}\right)$ and $P_{v}\left(\right.$ item $\left.\mid D i e_{2}\right)$ are $0.3,0.2,0.6$ and 0.7 , respectively. Then, the occurrence probability of the interest evidence $D i e_{1}$ for the entity item, i.e. $P_{v}\left(D_{i e_{1}} \mid\right.$ item $)$ can be calculated by,

$$
\begin{aligned}
P_{v}\left(\text { Die }_{1} \mid \text { item }\right) & =\frac{p\left(\text { Die }_{1}\right) \times P_{v}\left(\text { item } \mid \text { Die }_{1}\right)}{\left.P\left(\text { Die }_{1}\right) \times_{v}\left(\text { item } \text { Die }_{1}\right)+P\left(\text { Die }_{2}\right) \times P_{v}(\text { item }) \text { Die }_{2}\right)} \\
& =\frac{0.3 \times 0.6}{0.3 \times 0.6+0.2 \times 0.7}=0.5625
\end{aligned}
$$

Next, based on Equation (14), the explicit interest value $I(v \mid$ item $)$ can be calculated according to the above calculation results. For example, firstly, when the condition $n=0$ is true, the value of $I(v \mid$ item $)$ is zero. When the condition is true, to make the calculation easier, we assume that the influence of explicit interest $\lambda^{\text {impact }}$ is 0.8 , the value of $\operatorname{right}_{\varsigma_{i} \in D i e_{1}}\left(D_{i e_{1}}\right)$ is 0.6 and $n=1$. Then the explicit interest value about the user node $v$ to an entity item is calculated as follows:

$$
\begin{aligned}
I(v \mid \text { item }) & =\left[\frac{1}{1} \times\left(\operatorname{right}_{\varsigma_{i} \in \text { Die }_{1}}\left(\text { Die }_{1}\right) \times P_{v}\left(\text { Die }_{1} \mid \text { item }\right)\right)\right] \times \lambda^{\text {impact }} \\
& =\left[\frac{1}{1} \times(0.6 \times 0.5625)\right] \times 0.8=0.27 .
\end{aligned}
$$

To calculate the concrete weight of $\operatorname{right}\left(D i e_{j}\right)$, the inherent relationships between the interest evidence need to be considered, that is because some interest evidence may appear continuously and simultaneously. For example, the interest evidence of long time browsing is likely to happen simultaneously with the interest evidence "approving" or "add to favorite list", and they may influence each other 
and strengthen the contact. From this point of view, the basic principle of the weight calculation of interest evidence is similar to PageRank [35]. It means the more important interest evidence is likely to be associated with the other important interest evidence. In this study, the interest evidence $y$ caused by another interest evidence $x$ is denoted as: $x \rightarrow y$ for convenience. Therefore, the set that is associated with interest evidence $D_{i e_{i}}$ can be expressed as:

$$
L\left(D_{i e}\right)=\left\{D_{i} e_{j} \mid \exists\left(D_{i e_{j}} \rightarrow D_{i e}\right) \wedge(i \neq j)\right\} .
$$

Then, the weight of the interest evidence is calculated as follows:

$$
\operatorname{right}\left(D_{i} e_{i}\right)=\frac{1-p\left(D_{i e}\right)}{|I D|}+p\left(D_{i} e_{i}\right) \times \sum_{D_{i e_{j}} \in \operatorname{Link}\left(D_{i e}\right)} \frac{\operatorname{right}\left(D_{i} e_{j}\right)}{L\left(D_{i} e_{j}\right)}
$$

where $p\left(D i e_{i}\right)$ means the probability of interest evidence $D i e_{j}$ among the previous behaviors of the user, and $L\left(D i e_{j}\right)$ means the number of interest evidence that link with interest evidence $D i e_{j}$.

Since the user interest will change by time, we propose a dynamic prediction method based on the aging algorithm to describe the change of interest. Assume the explicit interest about the user $v$ to the entity at timestamp $t_{n-1}$ is $I(v \mid \text { item })_{n-1}$, the explicit interest about the user $v$ to the entity at the next timestamp $t_{n}$ is $I(v \mid \text { item })_{n}$. Then the predicted explicit interest at timestamp $t_{n}$ is based on $I(v \mid \text { item })_{n-1}$ and $I(v \mid \text { item })_{n}$. The calculation method is in Equation (18).

$$
I(v \mid \text { item })_{n}=\xi \times I(v \mid \text { item })_{n-1}+(1-\xi) \times \text { Change_I }{ }_{v}(\text { item })_{n} .
$$

At last, if the explicit interest degree of a user to an entity is greater than or equal to a given threshold $\beta$, where the value of $\beta$ is based on the experiment in Section 5 to obtain the optimal solution, then the user will be interested in this particular entity.

\subsection{Multi-Dimensional Comprehensive Recommendation Algorithm Based on Social Network}

In this study, a trust-based multi-dimensional comprehensive recommendation algorithm on social network is proposed, which mainly contains four algorithm modules, including the user candidate set algorithm, the entity candidate set algorithm, the user interest degree algorithm, and the comprehensive module recommendation algorithm. The four algorithms will be introduced in the following sub-sections, respectively.

\subsubsection{User Candidate Set Algorithm}

The model of the proposed recommendation method is based on trust between users on social network, and the assessment of trust is mainly based on the strength of 


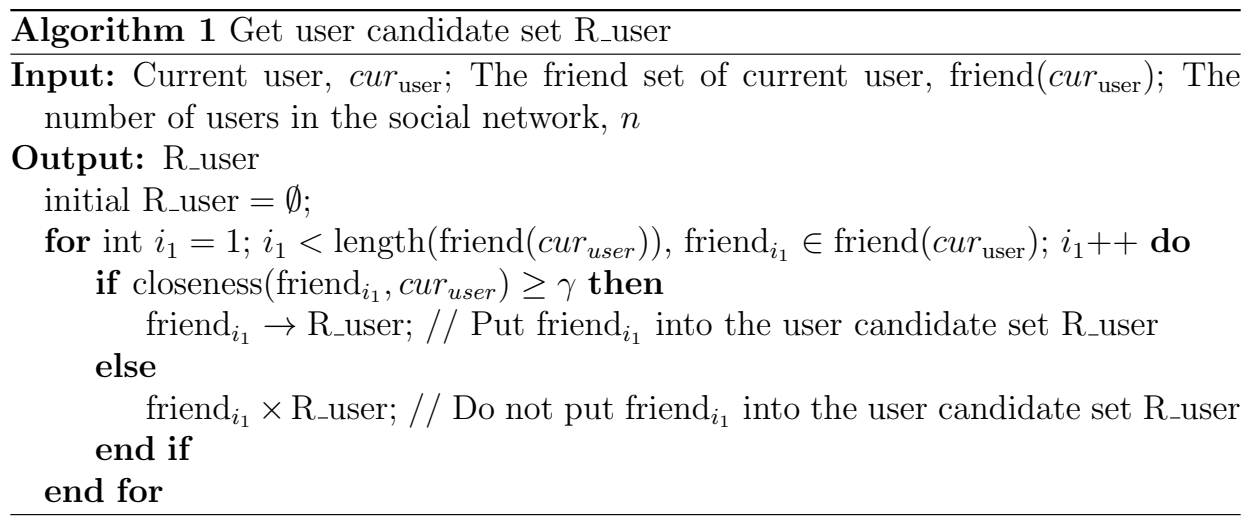

the user relationship. If a current user has a higher relationship strength with his (her) friend user, that means there is a greater similarity between them. Then, the purchased entities of the current user will be recommended to this particular friend user [36]. The ultimate goal of the user candidate set algorithm is to retrieve the user candidate set R_user for each current user $c u r_{\text {user }}$. This method can join the user candidate set with the friends who have a strong relationship with the current user. This process will inevitably include some new users, who may also have the recommended entity set corresponding to them, which can solve the cold start problem to some extent. The method in detail is introduced in Algorithm 1 . The time complexity of this algorithm is $O(n)$, where $n$ represents the number of users in the social network.

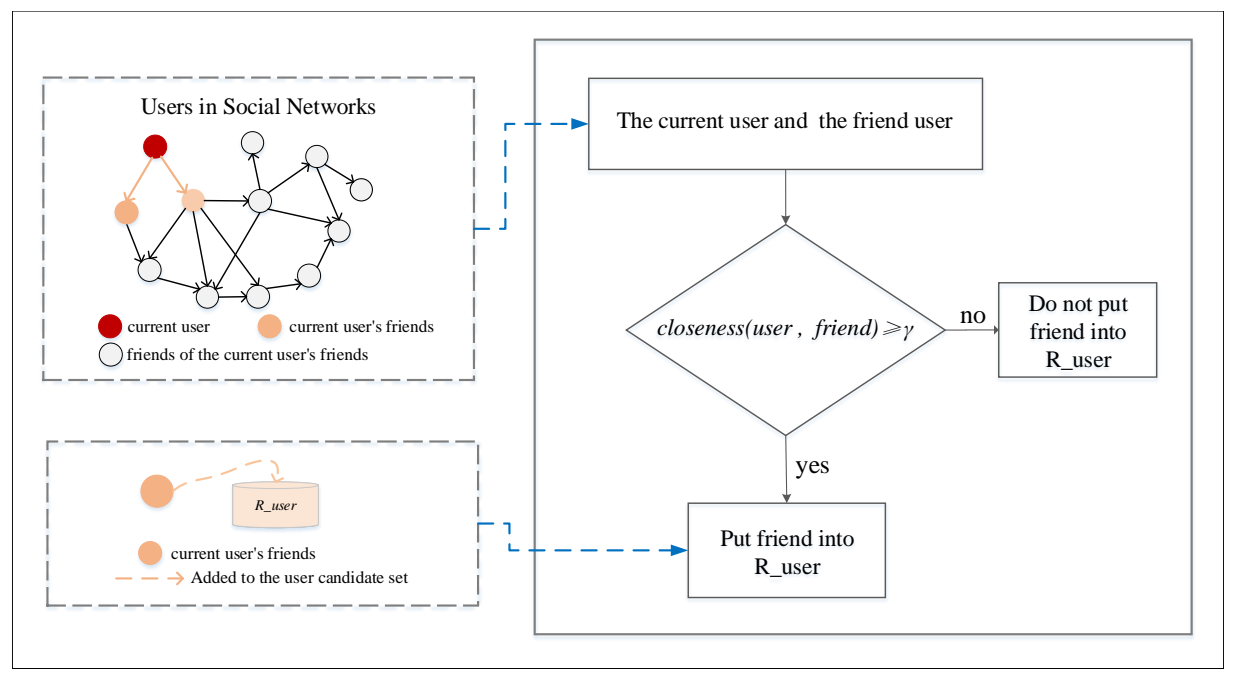

Figure 6 . The flow of user candidate set algorithm 
Figure 6 shows the general flow of Algorithm 1 On the left side of Figure 6, the red dot in social network circle represents the current user, and the orange dots that are directly connected with the current user represent the current user's friends, and the remaining gray dots represent the friends of the current user's friends. If the user relationship strength between the current user and the friend user is greater than or equal to the threshold $\gamma$, where $\gamma$ is set by the experiment in Section 5 as well, then the friend user will be added to the user candidate set R_user; otherwise, he (she) will not be added.

\subsubsection{Entity Candidate Set Algorithm}

Since the ultimate goal of the recommendation system is to recommend the corresponding entities to users, and the e-commerce websites contain a large number of entities, the entities that are similar to the purchased entity need to be chosen as the recommendation entities. In another word, the current user has purchased an entity $A$, then the entity $B$ that is similar to the entity $A$ will be recommended to the friend users who have similar preferences with the current user. Therefore, the entity candidate set R_item that contains similar entities with a particular entity needs to be obtained. The method in detail is shown in Algorithm 2. The time complexity of this algorithm is $(m \log m)$, where $m$ represents the number of entities in the initial entity candidate set.

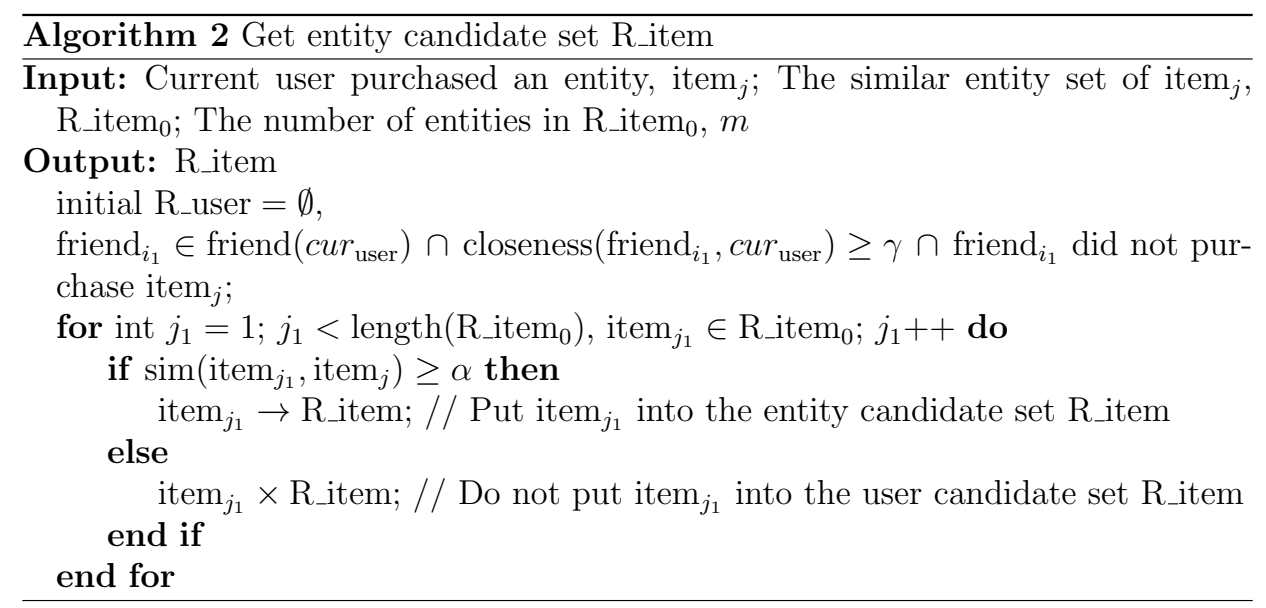

Figure 7 shows the general flow of Algorithm 2. In this figure, if the current user has purchased an entity item ${ }_{j}$, then each entity from the entity library will be selected to calculate the similarity with the entity item $_{j}$, if the similarity is bigger than or equal to the threshold $\alpha$, then this entity item $_{j_{1}}$ needs to be added to the entity candidate set R_item. 


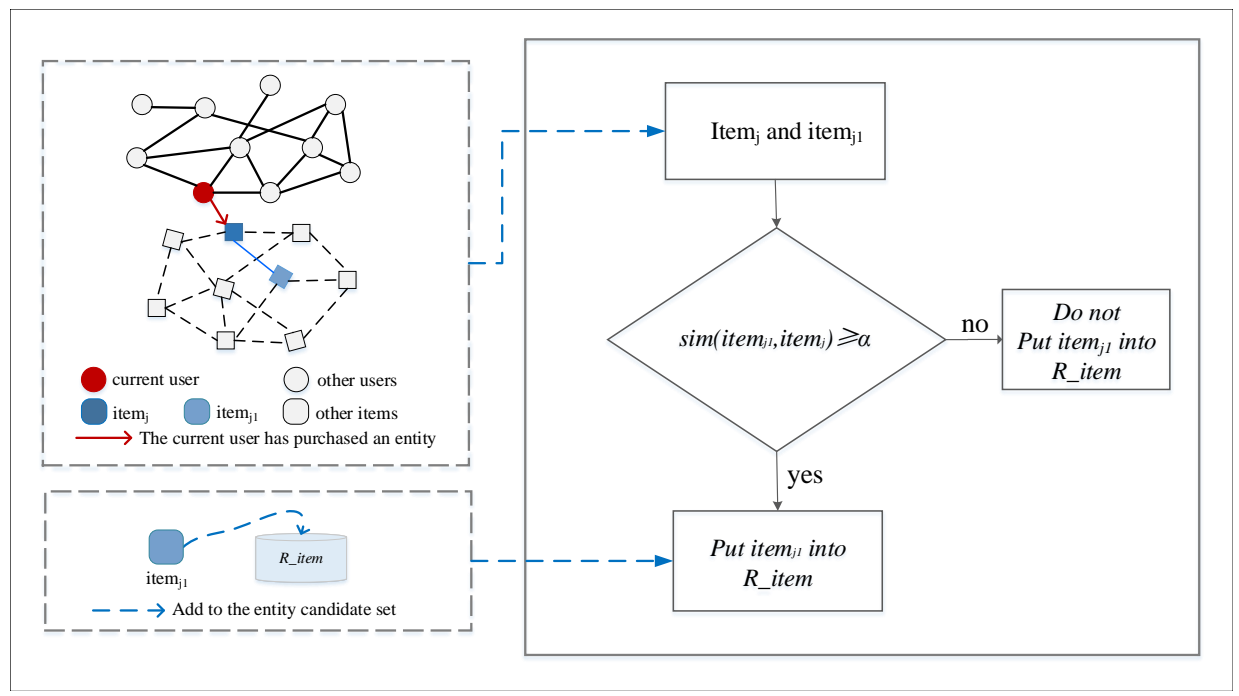

Figure 7. The flow of entity candidate set algorithm

\subsubsection{User Interest Algorithm}

The recommendation system concerns about if the user is really interested in the recommended entity, which can be measured by if a user purchased the recommended entity ultimately. In this study, we propose a user interest degree calculation method to decide whether to recommend an entity to the current user or not. The method is shown in Algorithm 3. The user interest $I$ (friend ${ }_{i_{2}} \mid$ item $_{j_{2}}$, item $_{j_{2}} \in$ R item) represents the interested degree of the user in the user candidate set $\mathrm{R}_{-}$user to the entity in the entity candidate set R_item. If the user interest degree is greater than or equal to the threshold $\beta$, then the entity item $_{j_{2}}$ will be recommended to the current user friend $i_{2}$. The time complexity of this algorithm is $(n * m \log m)$, where $n$ represents the number of users in the user candidate set and $m$ represents the number of entities in the entity candidate set $\mathrm{R}_{-}$item $_{0}$ of the current entity.

Figure 8 shows the general flow of Algorithm 3 . In this figure, first, each friend user friend $i_{i_{2}}$ is selected from the user candidate set $\mathrm{R}_{-}$user of the current user, and each entity is selected from the entity candidate set R_item that contains the entities purchased by the current user. Then, calculate the user interest degree of the user friend $i_{2}$ to the entity item $j_{j_{2}}$, denoted by $I\left(\right.$ friend $_{i_{2}} \mid$ item $\left._{j_{2}}\right)$, if the user interest degree is greater than or equal to the threshold $\beta$, then the entity item $_{j_{2}}$ will be recommended to the friend user friend $i_{i_{2}}$. 

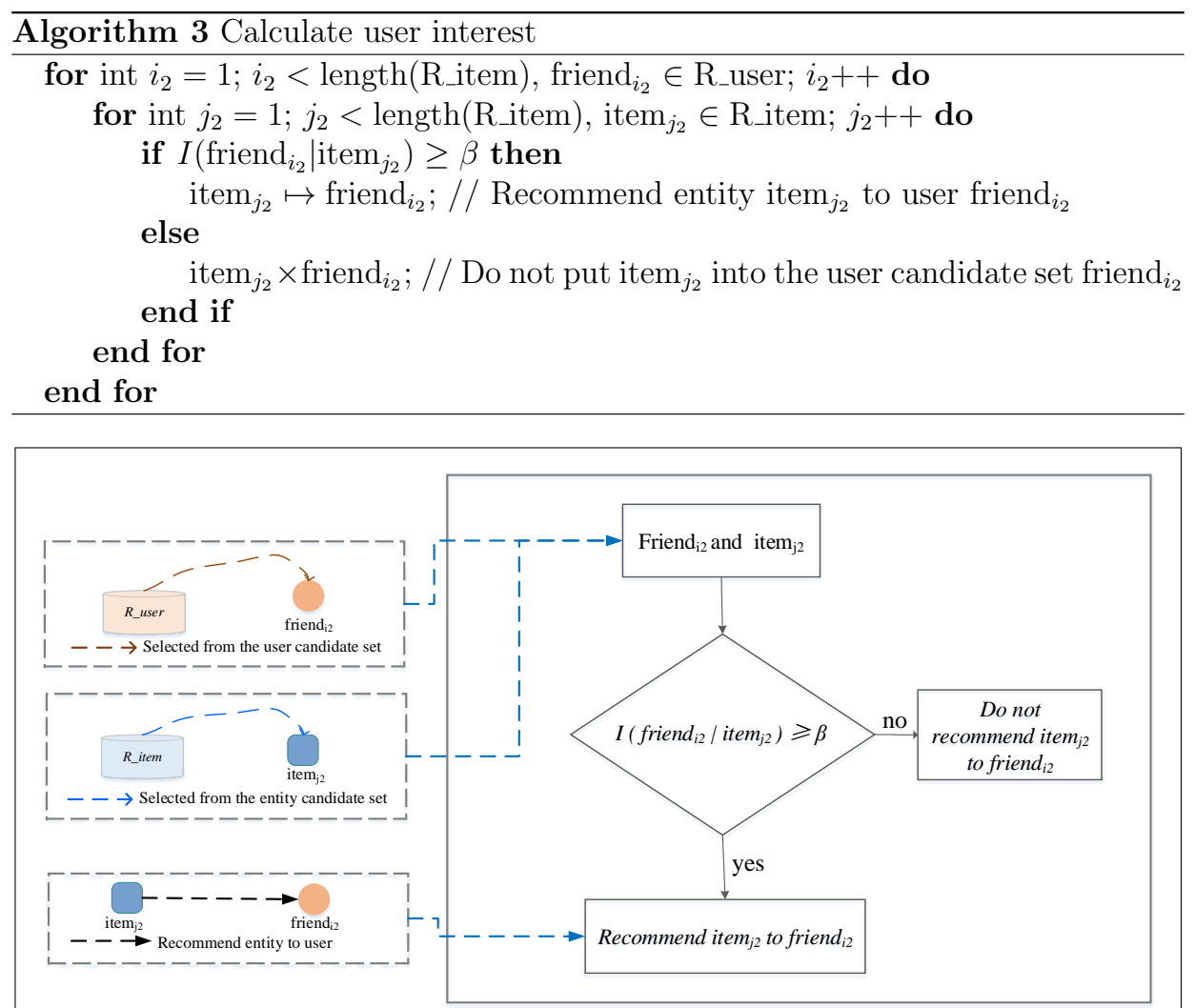

Figure 8 . The flow of the user interest algorithm

\subsubsection{The Comprehensive Module of the Recommendation Algorithm}

The previous three sub-sections describe the user candidate set algorithm, the entity candidate set algorithm and the user interest degree algorithm, respectively. These algorithms are mainly used for estimating the corresponding recommendation factors. Based on these factors, we will introduce a comprehensive module recommendation algorithm in this section. The main purpose of the algorithm is to retrieve the appropriate entity in the entity candidate set R_item, and recommend it to the user in the user candidate set R_user. The method in detail is shown in Algorithm 4 .

In this algorithm, the user relationship in social network is considered as a tree structure. The corresponding nodes in the tree structure can be considered as user nodes. First, based on the tree structure, calculate the user relationship strength between the user and the user's friend based on Algorithm 1. Second, calculate 
the entity similarity between the purchased entity and other entities in the entity candidate set based on Algorithm 2. Then, calculate the user interest degree about the user in the user candidate set for the entity in the entity candidate set based on Algorithm 3. At last, recommend the entity in the entity candidate set that satisfies the conditions to the user in the corresponding user candidate set. The time complexity of this algorithm is $O(n * m \log m)$, where $n$ represents the number of users in the user candidate set and $m$ represents the number of entities in the entity candidate set $\mathrm{R}_{-}$item $\mathrm{m}_{0}$ of the current entity.

Algorithm 4 in detail is described in the following, which is the core of the multi-dimensional comprehensive recommendation method under the social network environment.

If a user $a$ purchased an entity $\operatorname{item}_{j}$, then retrieve the friend set friend $(a)$ of the user $a$. If a user $b_{i}$ belongs to friend $(a)$ and the closeness between user $b_{i}$ and user $a$ is bigger than or equal to the threshold $\gamma$, at the same time, the user $b_{i}$ has not purchased the entity item ${ }_{j}$, then the following steps will be performed.

Step 1: Retrieve the candidate recommendation set R_item of the entity item ${ }_{j}$ (noting that R_item has already contained item $\left._{j}\right)$ by calculating $\operatorname{sim}\left(\right.$ item $_{k}$, item $\left._{j}\right)$. If the $\operatorname{sim}\left(\right.$ item $_{k}$, item $\left._{j}\right)$ is bigger than or equal to the threshold $\alpha$, then put the entity item $_{k}$ into the set $\mathrm{R} \_$item.

Step 2: For each user $b_{i}$ in friend $(a)$, calculate the user strength closeness $\left(b_{i}, a\right)$ between the user $b_{i}$ and $a$, if closeness $\left(b_{i}, a\right)$ is greater than or equal to $\gamma$, then put the user $b_{i}$ into the set $\mathrm{R}_{-}$user. After that, calculate the user interest degree $I\left(b_{i} \mid \operatorname{item}_{k} \in \mathrm{R} \_\right.$item $)$, if $I\left(b_{i} \mid \operatorname{item}_{k} \in \mathrm{R} \_\right.$item $)$is greater than or equal to the threshold $\beta$, then recommend the entity $\operatorname{item}_{k}$ to the user $b_{i}$.

Step 3: The nodes of all the recommended entities are marked as $C_{p}$, regarding $C_{p}$ as $a$. Then the recommended entity in the recommendation candidate set R_item from Step 1 is continued to be recommended to the user $a$ 's friends, then repeat Step 2. Finally, the recommendation algorithm will end until all the users have been traversed in the social network.

The recommendation algorithm process is shown in Figure 9. The left side of this figure shows the calculation process of the user candidate set and the entity candidate set in detail. The right side shows the user interest degree algorithm.

\section{EXPERIMENTS}

\subsection{Experimental Dataset}

To evaluate the performance of the multi-dimensional comprehensive recommendation algorithm based on social networks, we implemented some experiments using Douban reading dataset and Sina Weibo dataset. Douban reading is a website about reading books, which can recommend corresponding books to users. In our crawled 


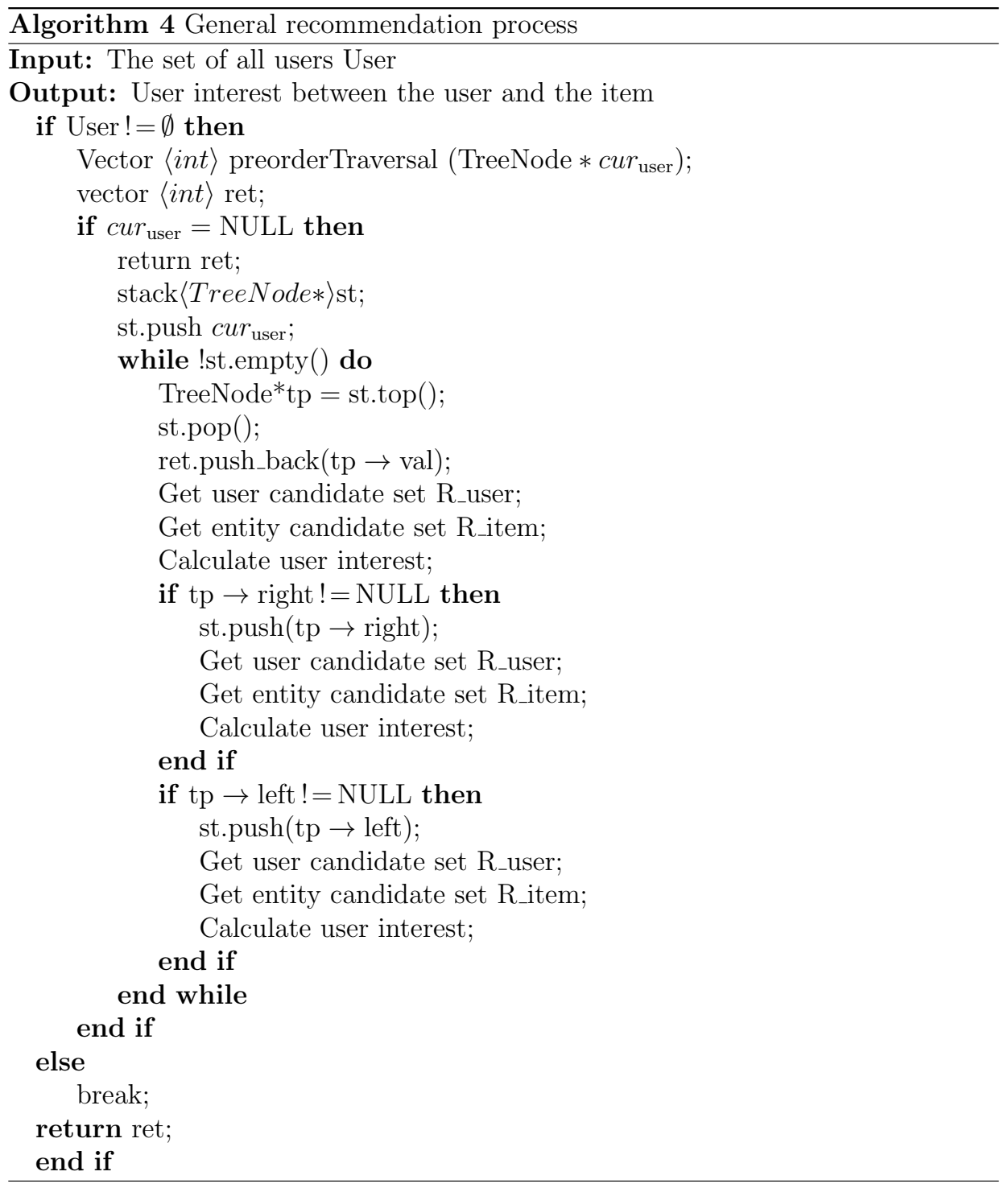

Douban reading data, it contains 55328 books in total, and each book contains corresponding information, including the serial number (a unique identifier corresponds to one book), book name, review score, price, category, retailer and user ID. Sina Weibo website is the largest micro-blogging site in China, which owns excellent social network features. In our crawled Sina Weibo data, it contains 63641 user records, and each user record contains information including user ID, user nickname, user's province, user's city, user's gender, the number of fans of the user, the number of 


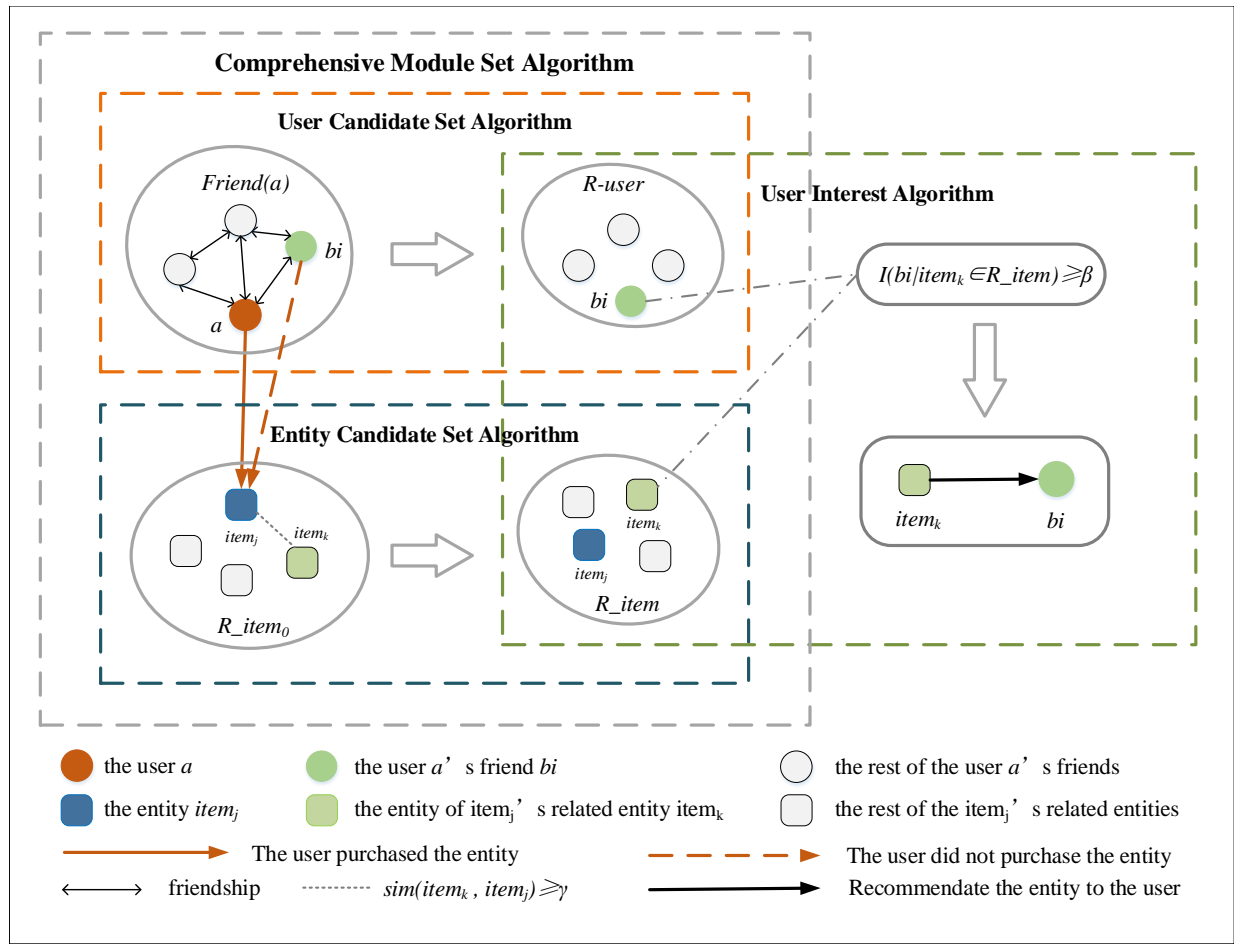

Figure 9. The overall process of the recommendation algorithm

friends of the user, the set of friends' ID of the users, the comments between friends and the number of interactions.

Since we proposed a multi-dimensional comprehensive recommendation system based on trust under the social network environment, the social network data and the e-commerce website data need to be linked. In this experiment, the fast login method, which means register an account in the commercial website by using the account in the social network, was chosen to solve this problem. Then the link between the user accounts in Sina Weibo and accounts in Douban reading can be established.

After retrieving user information and book information from Douban reading and Sina Weibo website, and establishing the user association between two datasets, we created a related table in Table 2. This table demonstrates the related data structure and description, which contains user account (userID), friend set account (friendsID), friend set number (friendsetNo), comment number (commentNo) and interaction frequency number (frequencyNo). To put it shortly, the friendsetNo represents the closeness between the current user and the current user's friends, the commentNo represents the comments between the current user and the current 
user's friends, and the frequencyNo indicates the interaction frequency between the current user and the current user's friends.

\begin{tabular}{|l|l|}
\hline Return Value Field & Field Description \\
\hline userID & User account \\
\hline friendID & Friend account \\
\hline friendsetNo & Friend set number \\
\hline commentNo & Comment number \\
\hline frequencyNo & Interaction frequency number \\
\hline
\end{tabular}

Table 2. Related data structure and description

Then, the experiment is implemented by the following four steps. First, the user's behavior dataset is divided into 8 parts randomly, one part is used as the test set and the remaining seven parts are used as the training set. Second, train the user interest model using the training set, and get the weights $\gamma_{1}, \gamma_{2}, \gamma_{3}$ and $\gamma_{4}$, which are required for estimating the strength of the user relationship, get the weights $\alpha_{1}, \alpha_{2}$, $\alpha_{3}$ and $\alpha_{4}$, which are required for estimating the similarity of the entities, and get the recommended thresholds $\alpha, \beta$ and $\gamma$ for each dimension based on the above results. Then, predict user behaviors on the test set using the thresholds retrieved from the

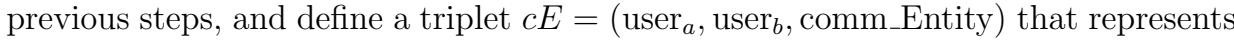

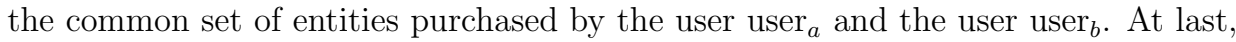
evaluate the prediction result on the test set by using some evaluation measurements that will be introduced in Section 5.2

\subsection{The Evaluation Metrics of the Recommendation Method}

In the experiment, three evaluation metrics were used to validate the performance of the recommendation algorithm, including precision, recall, and F1-score. By comparing the recommended items with the user's true selection records, we can calculate the evaluation metrics. The equation of precision is shown as follows:

$$
\text { precision }=\frac{\sum_{u \in U}|R(u) \cap B(u)|}{\sum_{u \in U}|R(u)|}
$$

where $R(u)$ is the recommendation list of each user according to the user behaviors in the training set, $B(u)$ is the behavior list of each user in the testing set. And the recall is calculated as follows:

$$
\text { recall }=\frac{\sum_{u \in U}|R(u) \cap B(u)|}{\sum_{u \in U}|B(u)|} .
$$

The F1-score is calculated based on precision and recall, which is shown in Equation (21).

$$
F_{1}=\frac{2 \times \text { precision } \times \text { recall }}{\text { precision }+ \text { recall }} .
$$




\subsection{Experimental Results and Analysis}

In this section, we will show our experimental results and analyze the results from three aspects, which are social network analysis, weight setting and experimental results.

\subsubsection{Social Network Analysis}

The proposed multi-dimensional comprehensive recommendation algorithm is based on user relationship strength in social network. In this section, we utilized Gephi tools [37] to draw the graph of user relationship, and then analyzed the relationship strength between users. The indegree of the user node represents the number of fans of the current user, and the outdegree of the user node represents the number of users that the current user followed.

In order to show the characteristics of the network topology, only a part of data is selected from the dataset for displaying in this experiment. Figure 10a) shows that the approximate distribution of user degrees in Sina Weibo dataset (there were 2347 user nodes and 5001 edges). The distribution of power obeys the power law distribution, which shows that the Sina Weibo dataset is a network topology with no scale characteristics. Figure $10 \mathrm{~b}$ ) shows the user relationship structure in social network, where each red dot represents a user. It is obvious that some users gathered to a cluster, which can indicate that these users belong to the same community. The average path length of the dataset selected in this study is 4.218 , which means the network structure conforms to the features of the small-world network. In summary, the network model constructed by the Sina Weibo dataset owns the scale-free and small-world characteristics. Therefore, it is proved that the selected dataset is effective for analysis.

\subsubsection{Weight Setting}

In this section, first, the four weights $\gamma_{1}, \gamma_{2}, \gamma_{3}$ and $\gamma_{4}$ are trained through experiments, which will be used to calculate the strength of user relationship. Second, the four weights $\alpha_{1}, \alpha_{2}, \alpha_{3}$ and $\alpha_{4}$ are trained to calculate the entity similarity. Third, the recommendation thresholds $\alpha, \beta$ and $\gamma$ are trained for each recommendation dimension. Finally, by using the thresholds and weights calculated above, we compare the performance of our proposed method with some traditional recommendation methods.

Firstly, since the source user's characteristic will affect the target user, the weights $\gamma_{1}, \gamma_{2}, \gamma_{3}$ and $\gamma_{4}$ that represent comment stability, mutual reliability, interactive frequency, and common neighbors and similar communities need to be considered comprehensively [38]. In this study, we make $\gamma_{1}=\frac{1}{n} \sum_{i=1}^{n} \gamma_{1 i}, \gamma_{2}=\frac{1}{n} \sum_{i=1}^{n} \gamma_{2 i}$, $\gamma_{3}=\frac{1}{n} \sum_{i=1}^{n} \gamma_{3 i}, \gamma_{4}=\frac{1}{n} \sum_{i=1}^{n} \gamma_{4 i}$, where $n$ denotes the number of all relevant user pairs in the Sina Weibo dataset, $\gamma_{1 i}, \gamma_{2 i}, \gamma_{3 i}$ and $\gamma_{4 i}$ indicate the comment stability, mutual reliability, interactive frequency, and common neighbors and similar com- 


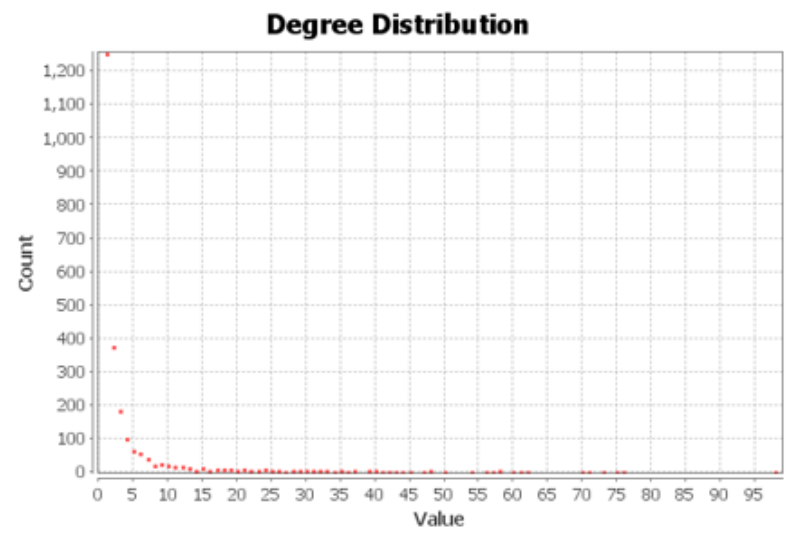

a)

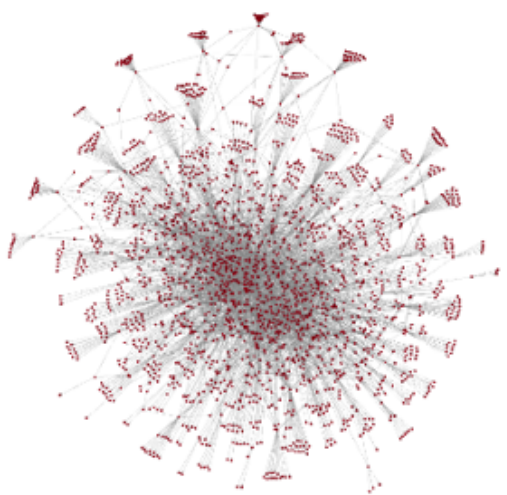

b)

Figure 10. a) The distribution of user degree, b) the structure diagram of social network

munities of the $i^{\text {th }}$ user group, respectively. In this study, the values of $\gamma_{1}, \gamma_{2}, \gamma_{3}$ and $\gamma_{4}$ are set to $0.10,0.40,0.25$ and 0.25 , shown in Table 3 , and the sum of $\gamma_{1}, \gamma_{2}$, $\gamma_{3}$ and $\gamma_{4}$ is 1 .

\begin{tabular}{l|l|r}
\hline Symbol & Description & Value \\
\hline$\gamma_{1}$ & the stability weights for comments between users & 0.10 \\
$\gamma_{2}$ & the weight of mutual reliability between users & 0.40 \\
$\gamma_{3}$ & the weight of interactive frequency between users & 0.25 \\
$\gamma_{4}$ & the weight of common neighbors and similar community & 0.25 \\
\hline
\end{tabular}

Table 3. The corresponding weights setting of user relationship strength 
Since the four weights will make different impacts on user relationship strength, the influence degree of each weight on the recommendation results needs to be examined. When the values of $\gamma_{1}, \gamma_{2}, \gamma_{3}$ and $\gamma_{4}$ are set to $0,0.40,0.25$ and 0.25 , respectively, the influence of the stability between users can be examined. When the values of $\gamma_{1}, \gamma_{2}, \gamma_{3}$ and $\gamma_{4}$ are set to $0.10,0,0.25$ and 0.25 , respectively, the influence of the mutual reliability between users can be examined. When the values of $\gamma_{1}, \gamma_{2}, \gamma_{3}$ and $\gamma_{4}$ are set to $0.10,0.40,0$ and 0.25 , respectively, the influence of the interactive frequency between users can be examined. And when the values of $\gamma_{1}, \gamma_{2}, \gamma_{3}$ and $\gamma_{4}$ are set to $0.10,0.40,0.25$ and 0 , respectively, the influence of the common neighbors and similar community can be examined.

Figure 11] shows the result of precision, recall, and F1-score by using different weights. The result of the original weights is shown in green color, which is clearly the best result. The precision, recall, and F1-score are the lowest when $\gamma_{2}$ is $0, \gamma_{1}$ is $0.1, \gamma_{3}$ is 0.25 and $\gamma_{4}$ is 0.25 , which is shown in light brown color. The precision, recall and F1-score results are about $23.2 \%, 23.3 \%$ and $23.4 \%$ lower than the original recommendation model. Therefore, compared with the other three factors, the mutual reliability has a greater impact on the strength of user relationship.
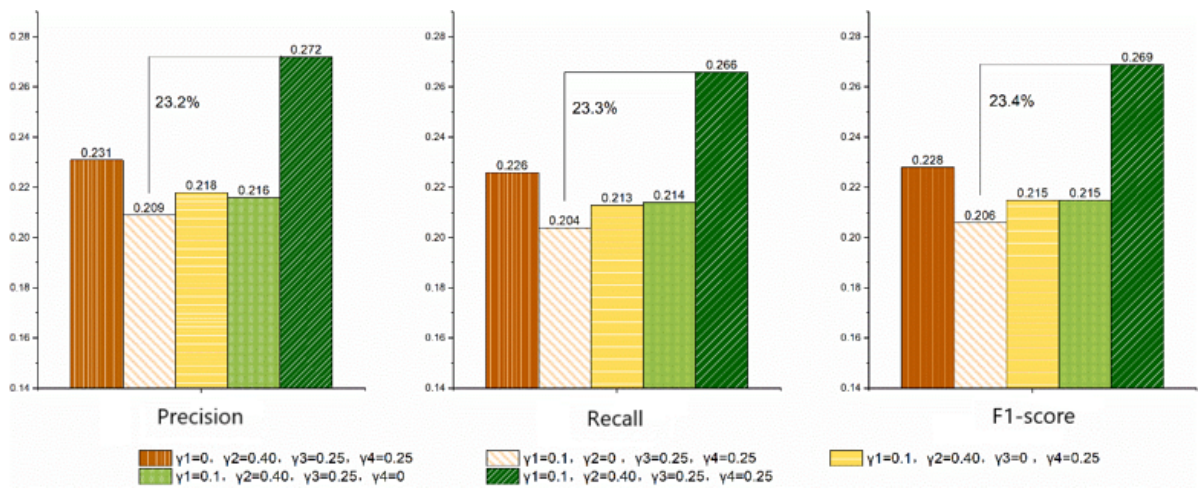

Figure 11. The results of using different $\gamma_{1}, \gamma_{2}, \gamma_{3}$ and $\gamma_{4}$

Secondly, the four weights $\alpha_{1}, \alpha_{2}, \alpha_{3}$ and $\alpha_{4}$ are required to calculate the entity similarity. Since both the category, price, comment, and sale of entities will affect the entity similarity to some extent, the values of $\alpha_{1}, \alpha_{2}, \alpha_{3}$ and $\alpha_{4}$ need to be considered comprehensively. In this study, we make $\alpha_{1}=\frac{1}{m} \sum_{i=1}^{m} \alpha_{1 i}, \alpha_{2}=\frac{1}{m} \sum_{i=1}^{m} \alpha_{2 i}, \alpha_{3}=$ $\frac{1}{m} \sum_{i=1}^{m} \alpha_{3 i}, \alpha_{4}=\frac{1}{m} \sum_{i=1}^{m} \alpha_{4 i}$, where $m$ denotes the number of all the relevant book pairs in Douban reading dataset, $\alpha_{1 i}, \alpha_{2 i}, \alpha_{3 i}$ and $\alpha_{4 i}$ indicate the category, price, comment, and sale of the $i^{\text {th }}$ books group, respectively. In this study, the values of the $\alpha_{1}, \alpha_{2}, \alpha_{3}$ and $\alpha_{4}$ are set to $0.35,0.28,0.27$ and 0.10 , respectively, shown in Table 4, and the sum of $\alpha_{1}, \alpha_{2}, \alpha_{3}$ and $\alpha_{4}$ is 1 .

Since the four weights will make different impacts on entity similarity, the influence degree of each weight on the recommendation results will be examined in this study as well. When the values of $\alpha_{1}, \alpha_{2}, \alpha_{3}$ and $\alpha_{4}$ are set to $0,0.28,0.27$ 


\begin{tabular}{l|l|r}
\hline Symbol & Description & Value \\
\hline$\alpha_{1}$ & category weight & 0.35 \\
$\alpha_{2}$ & price weight & 0.28 \\
$\alpha_{3}$ & comment weight & 0.27 \\
$\alpha_{4}$ & sale weight & 0.10 \\
\hline
\end{tabular}

Table 4. The corresponding weights setting of entity similarity

and 0.10 , respectively, the influence of the category weight can be examined. When the values of $\alpha_{1}, \alpha_{2}, \alpha_{3}$ and $\alpha_{4}$ are set to $0.35,0,0.27$ and 0.10 , respectively, the influence of the price weight can be examined. When the values of $\alpha_{1}, \alpha_{2}, \alpha_{3}$ and $\alpha_{4}$ are set to $0.35,0.28,0$ and 0.10 , respectively, the influence of the comment weight can be examined. And when the values of $\alpha_{1}, \alpha_{2}, \alpha_{3}$ and $\alpha_{4}$ are set to $0.35,0.28,0.27$ and 0 , respectively, the influence of the sale weight can be examined.

Figure 12 shows the result of precision, recall, and F1-score by using different weights of $\alpha_{1}, \alpha_{2}, \alpha_{3}$ and $\alpha_{4}$. The result shows that the original weight setting can achieve the best result, which is shown in dark green color. When $\alpha_{2}$ is $0, \alpha_{1}$ is $0.35, \alpha_{3}$ is 0.27 , and $\alpha_{4}$ is 0.10 , shown in dark brown color, the values of precision, recall, and F1-score are the lowest, which are approximately $21.7 \%, 21.8 \%$ and $21.9 \%$ lower than using the original weight. Therefore, compared with the other three factors, the category has a greater impact on entity similarity.

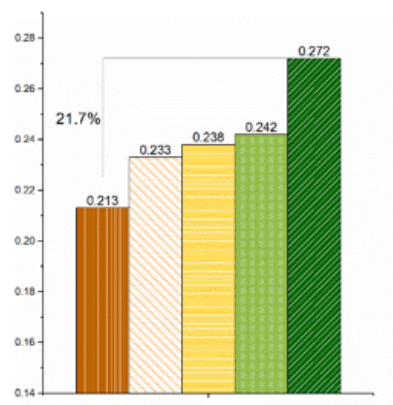

Precision

III] $a 1=0 . a 2=0.28, a 3=0.27, a 4=0.10$ $a 1=0.35, \quad a 2=0.28, \quad a 3=0.27, \quad a 4=0$

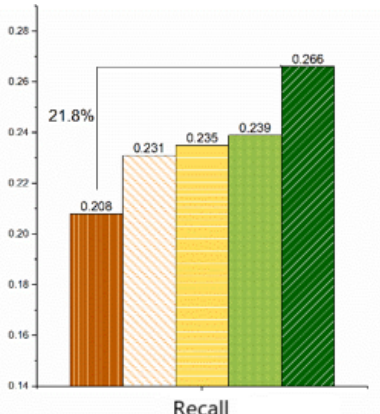

Recall

$\mathrm{a} 1=0.35, \quad \mathrm{a} 2=0, \mathrm{a} 3=0.27, \mathrm{a} 4=0.10$ $a 1=0.1, \quad a 2=0.40, a 3=0.25, \quad a 4=0.25$

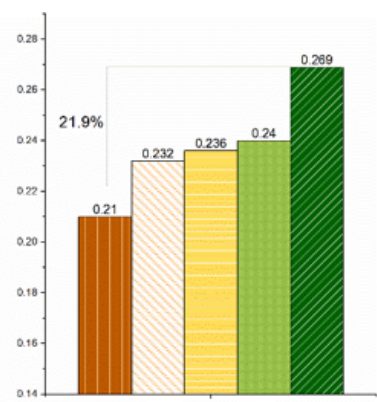

F1-score

$\square a 1=0.35, \alpha 2=0.28, \alpha 3=0, a 4=0.10$

Figure 12. The results of using different $\alpha_{1}, \alpha_{2}, \alpha_{3}$ and $\alpha_{4}$

At last, the results of using different recommendation thresholds $\alpha, \beta$ and $\gamma$ are shown in Table 5. The evaluation of the results is based on precision, recall and F1-score as well. When $\alpha$ is set to $0.3, \beta$ is set to 0.4 and $\gamma$ is set to 0.3 , emphasized in bold font, the proposed model can achieve the best performance. 


\begin{tabular}{l|r|r|r}
\hline$(\alpha, \beta, \gamma)$ & Precision & Recall & F1-score \\
\hline$(0.2,0.6,0.2)$ & 0.246 & 0.239 & 0.242 \\
$(0.3,0.5,0.2)$ & 0.249 & 0.245 & 0.247 \\
$(0.4,0.4,0.2)$ & 0.253 & 0.249 & 0.251 \\
$(0.5,0.3,0.2)$ & 0.258 & 0.256 & 0.257 \\
$(0.6,0.2,0.2)$ & 0.264 & 0.262 & 0.263 \\
\hline$(0.2,0.5,0.3)$ & 0.267 & 0.262 & 0.264 \\
$(\mathbf{0 . 3}, \mathbf{0 . 4}, \mathbf{0 . 3})$ & $\mathbf{0 . 2 7 2}$ & $\mathbf{0 . 2 6 6}$ & $\mathbf{0 . 2 6 9}$ \\
$(0.4,0.3,0.3)$ & 0.270 & 0.267 & 0.268 \\
$(0.5,0.2,0.3)$ & 0.263 & 0.261 & 0.262 \\
\hline$(0.2,0.4,0.4)$ & 0.261 & 0.259 & 0.260 \\
$(0.3,0.3,0.4)$ & 0.259 & 0.255 & 0.257 \\
$(0.4,0.2,0.4)$ & 0.257 & 0.252 & 0.254 \\
\hline$(0.2,0.3,0.5)$ & 0.256 & 0.252 & 0.254 \\
$(0.3,0.2,0.5)$ & 0.253 & 0.249 & 0.251 \\
\hline$(0.2,0.2,0.6)$ & 0.241 & 0.237 & 0.239 \\
\hline
\end{tabular}

Table 5. The values of Precision, Recall and F1-score for different $(\alpha, \beta, \gamma)$ pairs

\subsubsection{Experimental Results}

Finally, we compare our proposed method with some traditional recommendation methods using the same dataset. If our proposed method considers the entity similarity only, it will become the traditional content-based recommendation method; if it considers the strength of user relationship only, it will become the traditional social network-based recommendation method; if it considers the entity similarity and the user relationship strength only, it will become the knowledge-based recommendation method; and if the user relationship strength is not considered in our proposed method, it will become the traditional entity-based collaborative filtering recommendation method. In order to express conveniently, PNMCRS is used to represent our proposed method in this study, SNRS is used to represent the social network-based recommendation system method, KRS is used to represent the knowledge-based recommendation system method, CRS is used to represent the content-based recommendation system method, and CFRS is used to represent the collaborative filtering recommendation system method.

The comparison result is shown in Figure 13. It can be seen that our proposed comprehensive recommendation method has the highest accuracy, recall, and F1-score, which are $0.207,0.218$, and 0.212 , respectively, following by the social network-based recommendation method, which are $0.203,0.217$ and 0.210 , respectively. This is mainly because the traditional Pearson correlation coefficient (PCC) and Jaccard mean squared error (JMSD) are discarded when we calculate the user relationship strength in this study, however, the four aspects, which are comment stability, mutual reliability, interaction frequency, common neighbors and similar communities are taken into account. In general, the result proves that our proposed method can perform best when recommending entities to target users. 


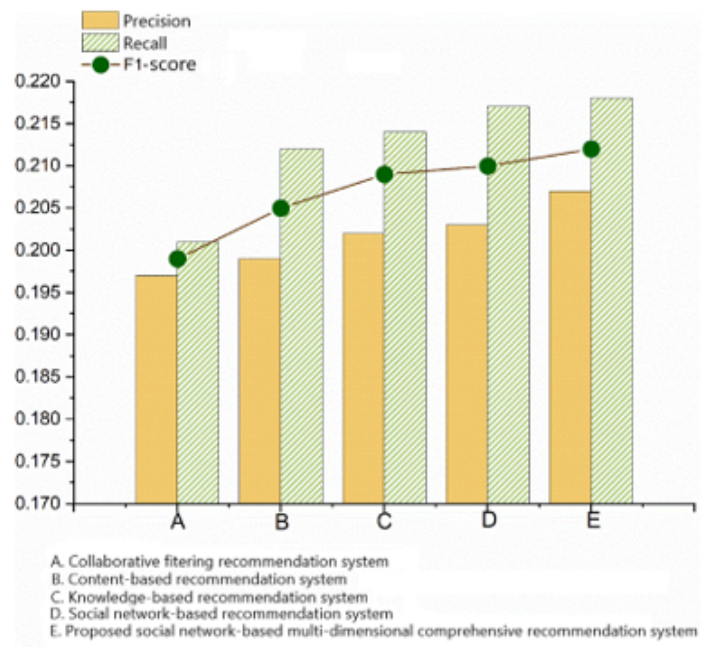

Figure 13. The three evaluation indicators of the proposed recommendation method and the traditional recommendation method

\section{CONCLUSION AND FUTURE WORK}

In social networks, mutual impact among users is common and inevitable. Improving recommendation performance from the perspective of user relationship strength is of great significance. In this paper, we propose a multi-dimensional recommendation algorithm from the perspective of user relationship strength in social network to improve the recommendation performance, which uses the user relationship, the similarity of entities and the degree of user interest information in three-level modeling comprehensively. In order to validate the effectiveness of our proposed model, we compared the performance of this novel model with some traditional recommendation models using the real-world dataset from Douban reading and Sina Weibo websites. The results of our experiments have demonstrated the excellent performance of our proposed model and its effectiveness on our existing dataset. The experimental results have been analyzed, which are consistent with the expected results. The proposed model can discover the interest degree of users and optimize the recommendation via multi-dimensional comprehensive recommendation factors based on user relationship strength in social network.

There are two major limitations in this study that could be addressed in future research. First, we ignore the behavior of some users who do not log in to the e-commerce platform through their social network accounts when implementing cross-platform data connection. In the future, we will retrieve more data to consider multiple login methods. Second, if the entity purchase time and information prop- 
agation time in social network can be considered in the recommendation algorithm as well, the accuracy may be further improved.

\section{Acknowledgement}

This work is funded by the National Natural Science Foundation of China (No. 61802258, No. 61572326, No. 61702333), the Natural Science Foundation of Shanghai (No. 18ZR1428300), the Shanghai Sailing Program (No. 19YF1436900), the Shanghai Committee of Science and Technology (No. 17070502800).

\section{REFERENCES}

[1] CAO, J.-Li, W.: Sentimental Feature Based Collaborative Filtering Recommendation. 2017 IEEE International Conference on Big Data and Smart Computing (BigComp), 2017, pp. 463-464, doi: 10.1109/BIGCOMP.2017.7881758.

[2] Li, S.-Luo, F.-YAng, J.-RAnzi, G.-Wen, J.: A Personalized Electricity Tariff Recommender System Based on Advanced Metering Infrastructure and Collaborative Filtering. International Journal of Electrical Power and Energy Systems, Vol. 113, 2019, pp. 403-410, doi: 10.1016/j.ijepes.2019.05.042.

[3] Liu, X.: A Collaborative Filtering Recommendation Algorithm Based on the Influence Sets of E-Learning Group's Behavior. Cluster Computing, Vol. 22, 2019, No. 2, pp. 2823-2833, doi: 10.1007/s10586-017-1560-6.

[4] Son, J.-Kim, S. B.: Content-Based Filtering for Recommendation Systems Using Multiattribute Networks. Expert Systems with Applications, Vol. 89, 2017, pp. 404-412, doi: 10.1016/j.eswa.2017.08.008.

[5] Shu, J.-Shen, X.-LiU, H.-Yi, B.-Zhang, Z.: A Content-Based Recommendation Algorithm for Learning Resources. Multimedia Systems, Vol. 24, 2018, No. 2, pp. 163-173, doi: $10.1007 /$ s00530-017-0539-8

[6] Suglia, A.-Greco, C.-Musto, C.-De Gemmis, M.-Lops, P.-Semeraro, G.: A Deep Architecture for Content-Based Recommendations Exploiting Recurrent Neural Networks. Proceedings of the $25^{\text {th }}$ Conference on User Modeling, Adaptation and Personalization (UMAP' '17), 2017, pp. 202-211, doi: 10.1145/3079628.3079684.

[7] Zhang, Y.-Saberi, M.-Chang, E.-Abbasi, A.: Solution and Reference Recommendation System Using Knowledge Fusion and Ranking. 2018 IEEE $15^{\text {th }}$ International Conference on E-Business Engineering (ICEBE), 2018, pp. 31-38, doi: 10.1109/ICEBE.2018.00016

[8] Guo, G.-Zhang, J.-Yorke-Smith, N.: Leveraging Multiviews of Trust and Similarity to Enhance Clustering-Based Recommender Systems. Knowledge-Based Systems, Vol. 74, 2015, pp. 14-27, doi: 10.1016/j.knosys.2014.10.016.

[9] Hong, Y.-Zeng, X.-Bruniaux, P.-Chen, Y.-Zhang, X.: Development of a New Knowledge-Based Fabric Recommendation System by Integrating the Collaborative Design Process and Multi-Criteria Decision Support. Textile Research Journal, Vol. 88, 2018, No. 23, pp. 2682-2698, doi: 10.1177/0040517517729383. 
[10] Resnick, P.-Varian, H. R.: Recommender Systems. Communications of the ACM, Vol. 40, 1997, No. 3, pp. 56-58, doi: 10.1145/245108.245121.

[11] Lu, X.-H.-Huang, H.-H.-Wu, H.-Y.-Liu, W.-L.: A Hybrid Recommendation Model for Community Attributes of Social Networks Based on Association Rule Mining. $20183^{\text {rd }}$ International Conference on Mechanical, Control and Computer Engineering (ICMCCE), IEEE, 2018, pp. 420-424, doi: 10.1109/ICMCCE.2018.00094.

[12] LI, M.-LI, Y.-Lou, W.-Chen, L.: A Hybrid Recommendation System for Q \& A Documents. Expert Systems with Applications, Vol. 144, 2020, Art. No. 113088, doi: $10.1016 /$ j.eswa.2019.113088.

[13] Puglisi, S.-Parra-Arnau, J.-Forné, J.-Rebollo-Monedero, D.: On Content-Based Recommendation and User Privacy in Social-Tagging Systems. Computer Standards and Interfaces, Vol. 41, 2015, pp. 17-27, doi: 10.1016/j.csi.2015.01.004

[14] Musto, C.-Semeraro, G.-De Gemmis, M.-Lops, P.: Learning Word Embeddings from Wikipedia for Content-Based Recommender Systems. In: Ferro, N. et al. (Eds.): Advances in Information Retrieval (ECIR 2016). Springer, Cham, Lecture Notes in Computer Science, Vol. 9626, 2016, pp. 729-734, doi: 10.1007/978-3-31930671-1_60

[15] Gu, Y.-Zhao, B.-Hardtke, D.-Sun, Y.: Learning Global Term Weights for Content-Based Recommender Systems. Proceedings of the $25^{\text {th }}$ International Conference on World Wide Web (WWW'16), 2016, pp. 391-400, doi: $10.1145 / 2872427.2883069$.

[16] Huang, H.-ZhaO, Q.: Social Book Recommendation Algorithm Based on Improved Collaborative Filtering. In: Liu, Q., Misir, M., Wang, X., Liu, W. (Eds.): The $8^{\text {th }}$ International Conference on Computer Engineering and Networks (CENet2018). Springer, Cham, Advances in Intelligent Systems and Computing, Vol. 905, 2018, pp. 477-484, doi: 10.1007/978-3-030-14680-1_52.

[17] Liu, S.-Dong, Y.-Chai, J.: Research of Personalized News Recommendation System Based on Hybrid Collaborative Filtering Algorithm. $20162^{\text {nd }}$ IEEE International Conference on Computer and Communications (ICCC), 2016, pp. 865-869, doi: 10.1109/CompComm.2016.7924826.

[18] Zhu, J.-Han, L.-Gou, Z.-Yuan, X.: A Fuzzy Clustering-Based Denoising Model for Evaluating Uncertainty in Collaborative Filtering Recommender Systems. Journal of the Association for Information Science and Technology, Vol. 69, 2018, No. 9, pp. 1109-1121, doi: 10.1002/asi.24036.

[19] Fang, Z.-GaO, S.-Li, B.-Li, J.-LiaO, J.: Cross-Domain Recommendation via Tag Matrix Transfer. 2015 IEEE International Conference on Data Mining Workshop (ICDMW), 2015, pp. 1235-1240, doi: 10.1109/ICDMW.2015.133.

[20] Du, Y.-Du, X.-HuAng, L.: Improve the Collaborative Filtering Recommender System Performance by Trust Network Construction. Chinese Journal of Electronics, Vol. 25, 2016, No. 3, pp. 418-423, doi: 10.1049/cje.2016.05.005. 
[21] Tarus, J. K.-Niu, Z.-Mustafa, G.: Knowledge-Based Recommendation: A Review of Ontology-Based Recommender Systems for E-Learning. Artificial Intelligence Review, Vol. 50, 2018, No. 1, pp. 21-48, doi: 10.1007/s10462-017-9539-5.

[22] Wang, D.-Xu, G.-Deng, S.: Music Recommendation via Heterogeneous Information Graph Embedding. 2017 International Joint Conference on Neural Networks (IJCNN), IEEE, 2017, pp. 596-603, doi: 10.1109/IJCNN.2017.7965907.

[23] Marwade, A.-Kumar, N.-Mundada, S.-Aghav, J.: Augmenting E-Commerce Product Recommendations by Analyzing Customer Personality. $20179^{\text {th }} \mathrm{In}-$ ternational Conference on Computational Intelligence and Communication Networks (CICN), IEEE, 2017, pp. 174-180, doi: 10.1109/CICN.2017.8319380.

[24] Kumar, S.- Varsha: Survey on Personalized Web Recommender System. International Journal of Information Engineering and Electronic Business (IJIEEB), Vol. 10, 2018, No. 4, pp. 33-40, doi: 10.5815/ijieeb.2018.04.05.

[25] Zhang, L.-Li, J.-Zhang, Q.-Meng, F.-Teng, W.: Domain KnowledgeBased Link Prediction in Customer-Product Bipartite Graph for Product Recommendation. International Journal of Information Technology and Decision Making, Vol. 18, 2019, No. 1, pp. 311-338, doi: 10.1142/S0219622018410031.

[26] Wang, H.-Zhang, P.-Lu, T.-Gu, H.-Gu, N.: Hybrid Recommendation Model Based on Incremental Collaborative Filtering and Content-Based Algorithms. 2017 IEEE $21^{\text {st }}$ International Conference on Computer Supported Cooperative Work in Design (CSCWD), 2017, pp. 337-342, doi: 10.1109/CSCWD.2017.8066717.

[27] Zhu, M.-Zhen, D.-Tao, R.-Shi, Y.-Feng, X.-Wang, Q.: Top-N Collaborative Filtering Recommendation Algorithm Based on Knowledge Graph Embedding. In: Uden, L., Ting, I. H., Corchado, J. (Eds.): Knowledge Management in Organizations (KMO 2019). Springer, Cham, Communications in Computer and Information Science, Vol. 1027, 2019, pp. 122-134, doi: 10.1007/978-3-030-21451-7_11

[28] Li, M.-Xiang, Y.-Zhang, B.-Huang, Z.-Zhang, J.: A Trust Evaluation Scheme for Complex Links in a Social Network: A Link Strength Perspective. Applied Intelligence, Vol. 44, 2016, No. 4, pp. 969-987, doi: 10.1007/s10489-015-0734-2

[29] Kalaï, A.-Zayani, C. A.-Amous, I.-Sedès, F.: Expertise and Trust-Aware Social Web Service Recommendation. In: Sheng, Q., Stroulia, E., Tata, S., Bhiri, S. (Eds.): Service-Oriented Computing (ICSOC 2016). Springer, Cham, Lecture Notes in Computer Science, Vol. 9936, 2016, pp. 517-533, doi: 10.1007/978-3-319-46295$0 \_32$.

[30] Hamid, M. N.-Naser, M. A.-Hasan, M. K.-Mahmud, H.: A Cohesion-Based Friend-Recommendation System. Social Network Analysis and Mining, Vol. 4, 2014, No. 1, Art. No. 176, doi: 10.1007/s13278-014-0176-6.

[31] Beigi, G.-Liu, H.: Similar but Different: Exploiting Users' Congruity for Recommendation Systems. In: Thomson, R., Dancy, C., Hyder, A., Bisgin, H. (Eds.): Social, Cultural, and Behavioral Modeling (SBP-BRiMS 2018). Springer, Cham, Lecture Notes in Computer Science, Vol. 10899, 2018, pp. 129-140, doi: 10.1007/978-3319-93372-6_15.

[32] Singh, H.: Defining and Delivering Personalized Entity Recommendations. September 26, 2019, US Patent Application, 15/935, 579. 
[33] Piao, G.-Breslin, J. G.: User Modeling on Twitter with WordNet Synsets and DBpedia Concepts for Personalized Recommendations. Proceedings of the $25^{\text {th }}$ ACM International on Conference on Information and Knowledge Management (CIKM '16), 2016, pp. 2057-2060, doi: 10.1145/2983323.2983908.

[34] Li, M.-Xiang, Y.-Zhang, B.-Wei, F.-Song, Q.: A Novel Organizing Scheme of Single Topic User Group Based on Trust Chain Model in Social Network. International Journal of Communication Systems, Vol. 31, 2018, No. 1, Art. No. e3387, doi: $10.1002 /$ dac.3387

[35] Lodigiani, C.-Melchiori, M.: A PageRank-Based Reputation Model for VGI Data. Procedia Computer Science, Vol. 98, 2016, pp. 566-571, doi: 10.1016/j.procs.2016.09.088.

[36] MA, X.-MA, J.-LI, H.-JiAnG, Q.-GaO, S.: ARMOR: A Trust-Based Privacy-Preserving Framework for Decentralized Friend Recommendation in Online Social Networks. Future Generation Computer Systems, Vol. 79, 2018, Part 1, pp. 82-94, doi: 10.1016/j.future.2017.09.060.

[37] Cherven, K.: Network Graph Analysis and Visualization with Gephi. Packt Publishing Ltd., 2013.

[38] Wang, Y.-Yin, G.-CAI, Z.-Dong, Y.-Dong, H.: A Trust-Based Probabilistic Recommendation Model for Social Networks. Journal of Network and Computer Applications, Vol. 55, 2015, pp. 59-67, doi: 10.1016/j.jnca.2015.04.007

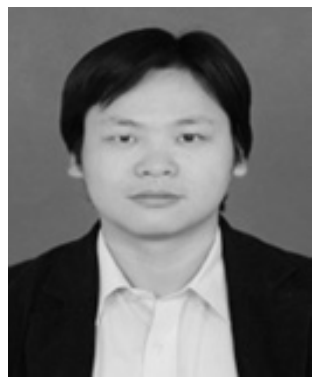

Bo ZHANG received his Ph.D. degree in computer science from the College of Electronics and Information Engineering, Tongji University, in 2009. And he finished his PostDoc research work in the Tongji University in 2012. He is now Professor in the College of Information, Mechanical and Electrical Engineering, Shanghai Normal University, Shanghai, China. His current research interests include trust computation and social network analysis. He is now Director of user group analysis project in social network, which is funded by the National Nature Science Foundation of China.

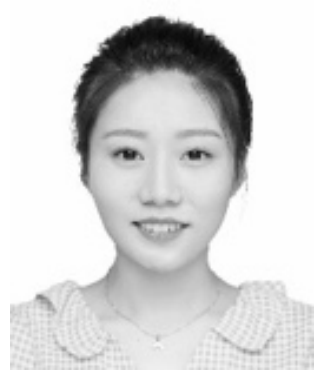

Ya ZhANG is Master's student in the College of Computer Science and Technology, Shanghai Normal University, China. She received her B.Sc. from the Shanghai Normal University in 2018. Her research interests are data mining, machine learning, and social network analysis. 


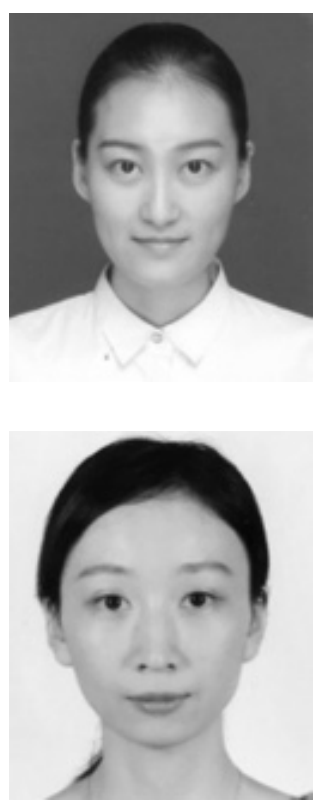

Yanhong BAI is now Master's degree candidate in computer application in the College of Information, Mechanical and Electrical Engineering, Shanghai Normal University. Her research interests include social network analysis, swarm intelligence, and group opinion dynamics in social networks.

Jie Lian is Assistant Professor in the Computer Science Department at the Shanghai Normal University where she has been a faculty member since 2017, and she obtained the "Sailing" Talent Program of China in 2019. She completed her doctoral degree at the Towson University in 2017. Her research interests are in the area of spatio-temporal data mining, deep learning and big data, ranging from the theory to design and implementation.

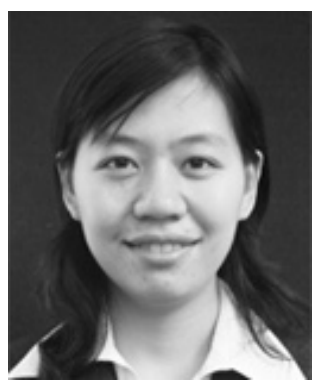

Meizi LI is now Associate Professor in the College of Information, Mechanical and Electrical Engineering, Shanghai Normal University, Shanghai, China. Her current research interests include social network analysis, trust and reputation computation. 